This item was submitted to Loughborough's Research Repository by the author.

Items in Figshare are protected by copyright, with all rights reserved, unless otherwise indicated.

\title{
BTZ-copolymer loaded graphene aerogel as new type Green and metal-free visible light photocatalyst
}

\section{PLEASE CITE THE PUBLISHED VERSION}

https://doi.org/10.1016/j.apcatb.2018.08.068

\section{PUBLISHER}

(C) Elsevier

\section{VERSION}

AM (Accepted Manuscript)

\section{PUBLISHER STATEMENT}

This paper was accepted for publication in the journal Applied Catalysis B: Environmental and the definitive published version is available at https://doi.org/10.1016/j.apcatb.2018.08.068.

\section{LICENCE}

CC BY-NC-ND 4.0

\section{REPOSITORY RECORD}

Tsang, Chi Him A., John Tobin, Jin Xuan, Filipe Vilela, Haibao Huang, and Dennis Y. Leung. 2018. "Btzcopolymer Loaded Graphene Aerogel as New Type Green and Metal-free Visible Light Photocatalyst”. figshare. https://hdl.handle.net/2134/35130. 


\section{BTZ-copolymer loaded Graphene Aerogel as New Type Green and Metal- Free Visible light Photocatalyst}

Chi Him A. Tsang ${ }^{\mathrm{a}, \mathrm{b}}$, John Tobin ${ }^{\mathrm{c}}$, Jin Xuan ${ }^{\mathrm{d}}$, Filipe Vilela ${ }^{\mathrm{c}}$, Haibao Huang ${ }^{\mathrm{a}^{*}}$, Dennis Y. C. Leung b* $^{*}$

${ }^{a}$ School of Environmental Sciences and Engineering, Sun Yat-Sen University, Guangzhou, PRC

${ }^{b}$ Department of Mechanical Engineering, The University of Hong Kong, Hong Kong

${ }^{c}$ School of Engineering and Physical Sciences, Heriot-Watt University, Edinburgh, Scotland, UK

${ }^{\mathrm{d}}$ Department of Chemical Engineering, Loughborough University, Loughborough, UL

*Corresponding authors, ycleung@hku.hk (DYCL), huanghb6@sysu.edu.cn (HH)

Abstract

This paper reports a new class of efficient, green and metal-free visible-light photocatalyst made from graphene aerogel (GA) doped with a conjugated porous polymer (CMPs). Hence, we report the synthesis of a benzothiadiazole (BTZ)-based CMP loaded into GA via a one-step hydrothermal reaction between $2 \mathrm{D}$ graphene oxide (GO) and the CMP, performed through a green process and under mild conditions. The as-prepared GA showed a bathochromic shift in the UV-visible diffraction reflectance spectroscopy (DRS) absorption edge to $628.5 \mathrm{~nm}$, demonstrating its ability to absorb light in the visible region. SEM, TEM, XPS, EDX mapping results further showed the successful loading of the BTZ-based CMP in the GA array. The synthesized GA was used as a 3D structured photocatalyst for the visible-light-driven photodecomposition of methyl orange (MO) with an efficiency of $89.2 \%$ (5 wt\% CMP). When compared to that of the pure CMP (86.9\%), a comparable yet small increase in the efficiency was observed. This is due to a synergistic effect between GO and loaded polymer in GA array upon the formation of CMPGA hybrid structure via chemical interaction between BTZ-Py and GO throughout the mild hydrothermal reaction, and the enhanced photocatalytic activity exhibited from $1 \mathrm{mg}$ equivalent polymer in the CMPGA2 hybrid when compared to the $20 \mathrm{mg}$ pure 
polymer. Upon repeated use, the depreciation in photocatalytic activity was low with a $<5 \%$ drop over 3 cycles. These results showed the CMP-loaded GA as an efficient metal-free photocatalyst and a promising material for further investigation into other photocatalytic applications.

Keywords: Graphene, visible light, photocatalyst, aerogel, conjugated polymer

\section{Introduction}

Photocatalysts are important materials for the degradation of environmental pollutants and green energy generation via solar energy conversion.[1-7] One issue with many photocatalysts today is the requirement for UV-light, which comprises about $5 \%$ of the total solar irradiation reaching the planet surface.[2] As a result, development of photocatalytic materials absorbing in the visible spectrum has become an important direction for innovation and sustainability.[2] A good photocatalyst has ability to prevent electron-hole $\left(\mathrm{e}^{-} / \mathrm{h}^{+}\right)$recombination upon light irradiation, allowing it to remain in an excited state. Since 3D structured nanoporous materials have larger surface areas compared to $2 \mathrm{D}, 1 \mathrm{D}$ and $0 \mathrm{D}$ nanomaterials due to multiple stacking of $2 \mathrm{D}$ nanomaterials sub-unit in the self-assembly process throughout the $3 \mathrm{D}$ structure formation. These properties are beneficial for charge transfer in a 3D nanomaterial array and prevents them from recombination, resulting in enhanced photocatalytic activity.[1]

Due to the large surface area provided by 3D structured porous materials and their associated properties of recombination inhibition, application of the above-mentioned materials in photocatalysis has become the major trend. In recent years, graphene aerogel (GA) based photocatalysts have been a new focus, exhibiting heightened activity for the photodecomposition of dyes.[2] To date, most of the GA-based photocatalysts are synthesized via three routes: $i$ ) 
simultaneous reduction of graphene oxide (GO) and metal ions into metal oxides; $[8,9]$ ii) direct reduction of metal oxides loaded $\mathrm{GO}[2]$; iii) and direct mixing of the photoactive materials with GO via hydrothermal reduction.[1] The conditions needed in some GA syntheses are relatively extreme, such as temperature requirements over $100{ }^{\circ} \mathrm{C}[1,3]$ or the use of toxic reducing agents like hydrazine.[2, 3] More recently, green preparations of GA-based photocatalysts under mild conditions have become an important avenue of research.[10,11] Moreover, as most research surrounding GA-based materials focuses on energy applications, investigations into their photocatalytic activity is relatively few.[1-5, 8, 12-15] This leaves a significant gap for further development into visible-light-driven GA-based photocatalysts.

In past decades, GA based photocatalysts absorbing in the visible spectrum were mainly composed of metal oxides as the loading materials.[2, 3, 8, 12, 13] However, recent developments in metal-free loading of GA have become prominent since they have the advantages over the extensively studied metal or metal oxide loaded GA photocatalysts, $[2,3,8$, $12,13]$ which included the free of metal and low cost of synthesis. Among them, $\mathrm{C}_{3} \mathrm{~N}_{4}[1,5,14$, 15] and dye molecules [4] were studied as immobilized photocatalysts within GA for visiblelight-driven photocatalytic reactions. Both materials showed strong activity in the photodegradation of organic dyes, $[1,14,15]$ and the photoreduction of $\mathrm{NO}_{\mathrm{x}},[5] \mathrm{CO}_{2},[1]$ nitroaromatic compounds,[4] and toxic metals.[4]

A class of polymers called conjugated microporous polymers (CMPs), first described by Cooper et al.,[16] have been developed as efficient metal-free photocatalytic materials in recent years.[17] Through careful design and selection of the monomers, these polymers can be tuned to absorb light in the visible spectrum. Among these materials, benzothiodiazole (BTZ)-based CMPs have been demonstrated as robust materials for energy applications[6] and visible-light- 
driven photocatalytic organic conversion (i.e. oxidation of $\alpha$-terpiene to ascaridole).[7] However, the use of CMPs as a metal-free photocatalytic additive to GA-based photocatalyst has not yet been reported. It is expected that a new type of metal-free GA-based photocatalyst can be generated with the combination of the nanoporous nature and effective charge transfer of GA and the low band gap properties of a BTZ-based CMP.

To date, most studies surrounding visible-light-driven GA photocatalysts have demonstrated strong activity in the photodecomposition of organic pollutants and dyes with efficiencies between $70-90 \% \cdot[1-5,8,9,12-15,18]$ In general, these reactions involve the use of fresh photocatalyst and started from a dark environment (i.e. 0th $\mathrm{min}$ ) with the purpose of achieving a dye/photocatalyst equilibrium mixture as the first step. The study continued from the dark equilibrium mixture until the end without refreshing the dye. $[1,3,5,8,9,12-15,18]$ However, this photocatalytic activity was calculated from the amount of the normalized dye concentration $\left(\mathrm{C} / \mathrm{C}_{0}\right)$ at the end of the reaction. $[1-5,8,9,12-15,18]$ Some of reactions were even carried out without the dark adsorption equilibrium throughout the photocatalytic reactions and no comparing with corresponding activity in dark condition.[2] Hence, use of such methods have risk of masking the actual activity of the photocatalyst due to the different initial concentration of the dye or reactants after the dark equilibrium process throughout the study, or the coexistence of adsorption and photocatalytic reactions. As a result, a modification of the study was carried out by using the dye or pollutant saturated materials as the photocatalyst under the light on reaction. It is because such practice is important for exploring the actual photocatalytic activity in the photodecomposition of the dye or pollutant through the use of saturated catalyst and the fresh dye together (i.e. using identical initial concentration). 
In this work, we demonstrate the synthesis of a 3D nanoporous structured CMP-loaded GA through a one-step hydrothermal reaction under mild conditions via the simultaneous assembly between a BTZ-based CMP and the GO nanosheets. In this instance, a BTZ-pyrene network (BTZ-Py) was chosen as the representative photocatalytic CMP (Fig 1). The synthesized GA product was named as a chemically modified polymer loaded graphene aerogel (CMPGA). The surface morphology and elemental information of CMPGA was analyzed in depth by various characterization methods. The photocataytic activity of CMPGA was evaluated via visible-lightdriven photodegradation of methyl orange (MO), one of the most popular organic dyes studied to demonstrate photocatalytic activity among GA-based photocatalysts.[1, 3, 8, 14, 15] The photocatalytic activity contributed from loading BTZ-Py in the GA array and the pre-saturation of the catalyst by MO via a dark absorption process prior to photodegradation reactions were also studied. These results showed that the metal-free CMPGA photocatalyst exhibited strong photocatalytic activity and good reusability, and the green and user-friendly synthesis methodology for the visible-light sensitive photocatalyst with strong activity. Interestingly, activity of demonstrated CMPGA with low BTZ-Py loading exhibited comparable activity to the pure CMP in the same reaction.

\section{Experimental}

\subsection{Materials}

Graphite powder (325 mesh), $\mathrm{P}_{2} \mathrm{O}_{5}$ (99\%, Sigma Aldrich), $\mathrm{K}_{2} \mathrm{~S}_{2} \mathrm{O}_{8}$ (99\%, Sigma-Aldrich), $\mathrm{KMnO}_{4}$ (99\%, Sigma-Aldrich), $\mathrm{H}_{2} \mathrm{O}_{2}$ (30\%, Sigma-Aldrich), sulphuric acid (98\%, SigmaAldrich), hydrochloric acid (37\%, Sigma-Aldrich), L-ascorbic acid (VC, 99\%, Sigma-Aldrich), DEPOT (99\%, Sigma-Aldrich), and methyl orange (MO, 99\%, Sigma-Aldrich) were used 
directly as purchased. The BTZ conjugated polymer (BTZ-Py) was synthesized similar to the work reported elsewhere,[7] the only difference is replacing the 1,3,5-triethynylbenzene by pyrene (Py) in the copolymer building unit for the BTZ-Py synthesis. The structure of BTZ-Py was illustrated in Fig 1.

\subsection{Synthesis of photosensitive polymer/GA composites}

The raw GO was synthesized from the modified Hummer's method which was reported elsewhere.[19] The as-prepared GO (90 mg) was then dispersed into DI water to obtain an aqueous dispersion of GO in an ultrasonic bath with a concentration of $3 \mathrm{mg} \mathrm{cm}^{-3} .36 \mathrm{mg}$ or 90 mg of BTZ-Py was then added to the graphene oxide/Tetrahydrofuran/water $\left(\mathrm{GO} / \mathrm{THF} / \mathrm{H}_{2} \mathrm{O}\right)$ dispersion and the resulting mixture was mixed with L-ascorbic acid (VC) $(0.75 \mathrm{~g})$ under an ultrasonic bath, followed by a stationary and mild conditioned chemical reduction at $60{ }^{\circ} \mathrm{C}$ for 48 h. The as-prepared structure was then cleaned by DI water through soaking for few days with DI water replacement for removing residual in the hydrogel $(\mathrm{GH})$. The cleaned hydrogel was then converted into aerogel (GA) under a freeze-drying process in a freeze dryer at $-80{ }^{\circ} \mathrm{C}$ under high vacuum for $48 \mathrm{~h}$. The GA obtained from BTZ-Py/GO was BTZ-Py/GA, named as CMPGA. Based on the EDX survey results obtained in Table 1, the name of sample obtained from the 36 mg BTZ-Py (2:5 mass ratio of BTZ-Py/GO) in the original dispersion was $2 \mathrm{wt} \% \mathrm{BTZ}-\mathrm{Py} / \mathrm{GA}$ and designated as CMPGA1, which was based on the sulphur content in the product. Similarly, the one from the $90 \mathrm{mg}$ BTZ-Py (1:1 mass ratio of BTZ-Py/GO) was $5 \mathrm{wt} \%$ BTZ-Py/GA and named as CMPGA2. We note that even though sulphur and nitrogen are distinct elements in the product (Fig 1), carbon exists in both BTZ-Py (Fig 1) and pure GO which was unsuitable to be 
used for loading percentage presentation in CMPGA. However, nitrogen cannot be detected in EDX analysis, sulphur was hence used for the loading percentage presentation.

\subsection{Photocatalytic activity in MO degradation}

Freeze-dried CMPGA samples with specific mass (10 or $20 \mathrm{mg}$ ) were mixed with $10 \mathrm{ppm}$ MO solution $(40 \mathrm{ml})$ under stirring at dark environment for the first $100 \mathrm{~min}$, followed by photocatalytic reaction for 80 min under irradiation of a visible-light source. A $300 \mathrm{~W}$ Xe lamp (Perfect-Light) equipped with a $420 \mathrm{~nm}$ filter was chosen as the light source, which had similar configuration to some other works.[1, 8,14$]$ Sample solution was taken out every $10 \mathrm{~min}$ and analyzed after centrifuge treatment to sink all the GA samples.

For comparison purpose, pure BTZ-Py and pure GA (10 and $20 \mathrm{mg})$ were also used in the MO reaction with the same operation parameters and light source. Self-degradation of MO was also carried out as another control sample. Fresh sample activity under full dark and full light environment was also carried out, $20 \mathrm{mg}$ of pure GA, CMPGA1 and CMPGA2 were used.

\subsection{Characterization}

The two as-prepared CMPGA samples were characterized by SEM (Hitachi S-4800 FEG SEM and FEI Quanta 400 FEG) and TEM (Philips FEI Tecnai G2 20 S-Twin STEM and Philips FEI Tecnai CM-100 TEM) to determine the material morphology, composition, and corresponding crystal structure. Elemental information of the CMPGA samples were analyzed by EDX installed in the SEM system and XPS (SKL-12 spectrometer modified with VG CLAM 4 multichannel hemispherical analyser and Thermo escalab 250Xi), respectively, where XPS signal peaks were calibrated with respect to sp2 carbon peak at $284.8 \mathrm{eV}$. The UV-visible diffraction reflectance 
spectroscopy (DRS) was carried out by a UV-vis spectrophotometer (Shimadzu, UV-2600) at wavelengths in the range of $200-800 \mathrm{~nm}$. The visible-light driven photocatalytic MO degradation performance of the CMPGAs was analyzed by the UV-vis spectrometer (METASH) at a characteristic wavelength of $464 \mathrm{~nm}$.

\section{Result and discussions}

\subsection{Materials characterization}

From the digital image of the bulk products obtained from the hydrothermal reduction as shown in Fig 2, black cylindrical blocks were observed, which were similar to pure GA reported in other works.[1,3] The morphology of the bulk sample synthesized from the hydrothermal reaction was characterized by the SEM as shown in Fig 3 with its EDX spectrum, of which the CMPGA2 was selected for presentation. Figs 3a-3d under different magnifications showed that graphene nanosheets in the product were stacked with each other after the hydrothermal reaction and freeze-drying process, which results in the formation of the nanoporous structures. The particles in the GA porous structures and the GNs surfaces in the CMPGA (Figs 3c-3d) seem to be the BTZ-Py polymer particles loaded in the GA products. Further analysis from the survey EDX spectrum (Fig 3e) showed the presence of carbon, oxygen, and sulphur with strong intensity, which partially come from the polymer and the GA background. By comparing the SEM images of pure GA (Figs 3f-3i) and BTZ-Py (Figs 3k-3n) under different magnification, pure GA was composed of nanosheets with nanoporous structure, while BTZ-Py was irregular particle and fiber structured polymer. In addition, corresponding EDX spectrum (Pure GA: Fig 3j and pure BTZ-Py: Fig 30) showed that the pure GA composed of carbon only due to the presence of a strong carbon peak. While pure BTZ composed of carbon, and sulphur due to 
presence of strong carbon and sulphur peaks. The presence of oxygen in GA was contributed from the residual oxygen in the reduced graphene oxide, while that in BTZ-Py was suspected to be arisen from the background rather than the sample itself. Since survey EDX spectrum pattern of CMPGA2 (Fig 3e) were laid between that of pure GA and BTZ-Py (Figs 3j and 3o), which showed that the elements detected in EDX of CMPGA (Fig 3e) was contributed from both GA and BTZ-Py. As a result, the particle like structure observed in CMPGA was totally contributed from the pure BTZ-py. This was the primary evidence of the loading of the BTZ-Py into the GA array through the hydrothermal reduction of GO into GA.

Since the normal EDX survey cannot totally reflect the true picture of the structure, the full frame EDX mapping characterization was then carried out as shown in Fig 4 with the comparison of the corresponding SEM images of the CMPGA samples, pure GA and pure BTZPy. The EDX mapping results of the CMPGA1 showed that the mapping of C-Ka (Fig 4a) and $\mathrm{O}-\mathrm{K} \alpha$ (Fig 4c) was very clear in intensity, while that of S-Ka (Fig 4e) was not strong enough. However, the patterns were still matched with the corresponding SEM image (Fig 4g). Similar results were observed in the CMPGA2 (Figs 4b, 4d, 4f and 4h), but the intensity of S-K $\alpha$ (Fig 4f) was stronger than that in the CMPGA1. This reflected that the larger the amount of the pure BTZ-Py added to the GO dispersion $\left(3 \mathrm{mg} \mathrm{ml}^{-1}\right)$, the larger the amount of the polymer loaded into the GA array, which highly matched with the EDX survey results listed in Table 1. In contrast, the EDX mapping of pure GA and BTZ-Py shown in Figs 4i-4p showed that only C-K $\alpha$ mapping pattern (Fig 4i) highly matched to the corresponding SEM image (Fig 41), but its O-Ka and $\mathrm{S}-\mathrm{K} \alpha$ patterns (Figs $4 \mathrm{j}-\mathrm{k}$ ) were relatively weak or even could not be observed. While in BTZ-Py (Figs 4m-4p), only C-Ka (Fig 4m) and S-Ka (Fig 4o) patterns matched with its pattern in corresponding SEM image of BTZ-Py (Fig 4p), but O-K $\alpha$ pattern (Fig 4n) observed were 
contributed from background instead of BTZ-Py sample (Fig 4p) due to the different pattern as compared with the C-K $\alpha$ and S-K $\alpha$ patterns. Comparing the EDX element mapping patterns of pure GA and BTZ-Py as shown in (Figs 4i-k, 4m-o), showed that composition of the CMPGAs matched that of BTZ-Py for S-K $\alpha$ part, while C-K $\alpha$ pattern matched to that of pure GA. These results reflected that $(i)$ the graphene obtained from CMPGAs were reduced graphene in nature after the mild hydrothermal reduction; and (ii) BTZ-Py were loaded into the GA array throughout the self-assembly of GO nanosheet step in VC assisted CMPGA formation.

Besides the morphological analysis performed by SEM, TEM was also carried out to observe further details of the CMPGA. The TEM images obtained in Fig 5 with different magnifications clearly showed the structure of the pure graphene nanosheets in the BTZ-Py loaded graphene. However, large irregular shaped blocks appeared in the sample, which may contribute from the presence of the BTZ-Py clusters. Comparing the TEM images of CMPGA with pure GA (Figs 5e-h) and BTZ-Py (Figs 5i-1) under different magnifications showed that pure GA composed of purely reduced graphene nanosheets, and BTZ-Py was irregular shaped amorphous polymer. Such findings showed that the morphology of the small irregular pieces and the nanosheets in the CMPGA (Figs 5a-5d) were similar to the pure BTZ-Py and graphene nanosheets in pure GA. It further proved that CMPGA composed of both graphene nanosheets from the GA background and irregular fragments of BTZ-Py after the ultrasonic treatment before the CMPGA synthesis.

Since elemental mapping of the samples from the EDX analysis and the SEM/TEM images cannot totally reflect the elemental status and whether loading of polymer to the GA has been taken place or not, characterization via XPS becomes an important method to compensate the weakness of EDX mapping. Figs $6 \mathrm{a}$ and $6 \mathrm{~b}$ showed the XPS survey spectra of the CMPGA1 and CMPGA2 respectively, which showed the presence of carbon, nitrogen, and sulphur in the 
samples. In contrast, only carbon, and oxygen signal peaks were observed in the survey spectrum of pure GA (Fig 6c), while strong carbon, nitrogen and sulphur signal peaks were observed in pure BTZ-Py (Fig 6d). The presence of nitrogen $(\sim 400 \mathrm{eV})$ and weak sulphur $(\sim 168 \mathrm{eV})$ peaks in Figs $6 a-6 b$ showed that the survey XPS pattern of CMPGAs were laid between that of pure GA and BTZ-Py, and close to that of BTZ-Py. It is primary evidence for the successful loading of BTZ-Py in GA. Further characterization by the HR-XPS showed that there was only single carbon, nitrogen, and sulphur peak observed in the C1s, N1s, and S2p spectra (Figs 6e-6j). It showed that the pristine carbon $\left(\mathrm{C}-\mathrm{C}\left(\mathrm{sp}^{2}\right): 284.8 \mathrm{eV}\right)$, oxidized carbon $(-\mathrm{C}-\mathrm{O}-\mathrm{x}: 286.05 \mathrm{eV})$, quaternary-nitrogen $\left(\mathrm{N}_{\mathrm{Q}}\right.$ : 399.7-399.8 eV), graphitic nitrogen $(402.1-402.5 \mathrm{eV})$, and sulphur (S2p1/2: 165.3-165.4 and C-S-C $167 \mathrm{eV}, 168.7-168.9 \mathrm{eV}$ ) were the major component in CMPGA1 and CMPGA2. Pristine carbon was originated from the reduction of the GO into GNs by VC during the GA formation, while the S peak was composed of multiple peaks at 165.4, 167.0, $168.7 \mathrm{eV}$ in CMPGA1 and 165.3, $168.9 \mathrm{eV}$ in CMPGA2, respectively. The HR-XPS recorded from pure GA (Figs 6k-6m) and pure BTZ-Py (Figs 6n-6p) showed that only obvious carbon signal was observed in C1s spectrum of pure GA (Fig 6k), and carbon, nitrogen, and sulphur peak were observed in C1s, N1s and S2p spectra of pure BTZ-Py (Figs 6n-6p). These peaks reflect the presence of pristine carbon $\left(\mathrm{C}-\mathrm{C}\left(\mathrm{sp}^{2}\right): 284.8 \mathrm{eV}\right)$, carbon-nitrogen bond (-C-N-: $285.71 \mathrm{eV})$, oxidized carbon (-C-O-: $286.05 \mathrm{eV})$, quaternary-nitrogen $\left(\mathrm{N}_{\mathrm{Q}}: 399.7 \mathrm{eV}\right)$, graphitic nitrogen $(401.8 \mathrm{eV})$, and sulphur (S2p1/2: 165.6, and C-S-C: 166.6-171.1 eV) in 2 separate pure samples. The pattern of N1s and S2p in CMPGAs (Figs 6f-6g, 6i-6j) were highly similar to that of pure BTZ-Py (Figs 6o-6p) due to the presence of $\mathrm{N}_{\mathrm{Q}}$ at 399.7-399.8 eV and graphitic nitrogen at 402.1-402.5 eV in N1s; and S2p1/2 at 165.3-165.4 eV and C-S-C at $167 \mathrm{eV}, 168.7-168.9 \mathrm{eV}$ in S2p. While C1s (Figs 6e and 6h) pattern was similar to that of pure GA (Fig 6k) according to 
the presences of C-C $\left(\mathrm{sp}^{2}\right)$ at $284.8 \mathrm{eV}$ and $-\mathrm{C}-\mathrm{O}-$ peak in same position of $286.05 \mathrm{eV}$. It primary showed that the composition and structure of CMPGAs was laid between that of pure GA and BTZ-Py. Even though weak N1s and S2p signals were detected in pure GA (Figs 61-m), the spectral peak pattern of N1s (Fig 61) and S2p (6m) in GA contributed to $\mathrm{N}_{\mathrm{Q}}(400.55 \mathrm{eV})$ and nitrate $(407.75 \mathrm{eV})$; and S 2p3/2 $(164.25 \mathrm{eV})$ and C-S-C $(168.8 \mathrm{eV})$, which was different from those of pure CMPGAs and BTZ-Py. Together with the zero wt $\%$ and at $\%$ of $S$ from EDX results (Table 1, Fig 4k) and no $\mathrm{S}$ and $\mathrm{N}$ signal observed in survey spectrum of GA (Fig 6c), N and $\mathrm{S}$ in the HR-XPS of GA were contributed from the background. In comparison with the survey EDX results (Figs 3e, 3j, 3o), even though N1s was observed in the corresponding XPS spectra, absence of $\mathrm{N}$ peak in the EDX analysis reflected the reason of using wt $\%$ of $\mathrm{S}$ for the presentation of BTZ-Py loading percentage in CMPGA synthesized in the current work.

In the in-depth interpretation of HR-XPS results of C1s and S2p of CMPGA2, an obvious resolved multiple peak observed in S2p of CMPGA1 (S 2p1/2: $165.4 \mathrm{eV}$, C-S-C: 167.0, 168.7 eV, Fig 6g) and CMPGA2 (S 2p1/2: $165.3 \mathrm{eV}, \mathrm{C}-\mathrm{S}-\mathrm{C}$ : $168.9 \mathrm{eV}$, Fig 6j) with extremely weak hidden C-S shoulder at $283 \mathrm{eV}$ of C1s spectrum of CMPGA2 (Fig 6h). Even though no obvious C-N $(285.8 \mathrm{eV})$, and C-S $(283 \mathrm{eV})$ signal was observed in the CMPGA samples (Figs 6e and 6h) when compared to pure BTZ-Py (Fig 6n) and other N or S-doped graphene products,[20-22] mentioned finding still showed the presence of C-S bonding when compared with literature had $\mathrm{C}-\left(\mathrm{SO}_{\mathrm{x}}\right)-\mathrm{C}$ structure.[21-24] This probably arose from the spin-orbit coupling between $\mathrm{S}$ and carbon in the CMPGA.[21, 22] Comparing with the corresponding pure components (GA (Fig 6m) and BTZ-Py (Fig 6p)), and other S-N doped GAs, [21-24] presence of S(0) 2p 1/2 and C-S-C peak in S2p spectra (Figs $6 \mathrm{~g}$ and $6 \mathrm{j}$ ) of CMPGAs was similar to the those recorded in the similar sample synthesized in other works.[21-24] Throughout comparing the amount of S in similar 
materials via different quantitative analysis in terms of atomic percentage at $\%$, total amount of $\mathrm{S}$ was $\sim 0.81-2 \%$ in CMPGAs which reflected from results in Table 1 . It was laid between the $\mathrm{S}$ content in the GA product recorded in similar works $(\sim 0.89-5$ at\%).[23, 24] Since HR-XPS spectra of S2p recorded in CMPGAs (Figs $6 \mathrm{~g}$ and $6 \mathrm{j}$ ) were similar to the case of relatively weak S2p HR-XPS pattern of NS-GA-2 with similar resolve C-S-C peak intensity observed from other reports.[23, 24] It primary interpreted that weak S 2p 1/2 finger print peak at $165 \mathrm{eV}$ in Figs $6 \mathrm{~g}$ and $6 \mathrm{j}$ may be reflected from low polymer loading in the GA array as mentioned in Table 1 , and strong interaction between $\mathrm{S}$ and $\mathrm{C}$ in BTZ-Py and $\mathrm{GO}$ via $\mathrm{C}-\left(\mathrm{SO}_{\mathrm{x}}\right)-\mathrm{C}$ bond formation throughout the CMPGA synthesis. In addition to the similar N1s pattern in CMPGAs and pure BTZ-Py, which reflecting the C-N bond from the BTZ-Py molecule (Fig 1) by the presence of graphitic N peak (Figs 6f, 6i, 6o). It showed that covalent interaction was formed between carbon in graphene and sulphur in polymer molecule during the hydrothermal reaction. While remaining part of the copolymer molecule exhibited non-covalent $\pi$ - $\pi$ interaction with graphene background in CMPGA. Such finding was similar to some of the N-doped GA and S-doped GA based products.[21, 25] This point showed that the nature of the polymer was not changed by the addition of VC during the hydrothermal reduction.

Besides the structural information of the CMPGA, its optical property was another target of interest. Fig 7 showed the UV-vis DRS spectrum of the CMPGA, samples were compared with that of pure BTZ-Py and pure GA. No peak and absorption edge was observed in the pure GA, while the absorption edge at $588 \mathrm{~nm}$ starts appeared in the CMPGA when the weight percentage of BTZ-Py was 2 wt\% (Fig 7b). Further increase in the BTZ-Py content (5 wt \%) enhanced the absorption peak intensity which was as clear as that of the pure BTZ-Py composite (Fig 7b), even though the intensity was lower than that of pure BTZ-Py (Fig 7a). Such finding showed that 
optical properties of the composite may be beneficial to the photochemical activity, which also reflected from red shift of absorption edge from $588 \mathrm{~nm}$ in CMPGA1 to $628.5 \mathrm{~nm}$ in CMPGA2. By comparison of absorption edge of pure BTZ-Py $(789 \mathrm{~nm})$ with CMPGAs in Fig 7a, the blue shift of absorption edge in CMPGA products showed that visible-light absorption was enhanced via combining BTZ-Py with GO throughout the CMPGA synthesis.

Up to date, most of the visible-light sensitive GA based products were synthesized using toxic reducing agents like cetyltrimethyl ammonium bromide (CTAB) and dopamine (DA).[3, 14, 18] Such products also produced toxic residual after the synthesis. In our present study, a green reaction was adopted with the use of the non-toxic reducing agent $\mathrm{VC}$ with a low reaction temperature of $60{ }^{\circ} \mathrm{C}$, and similar bulk product appearance was achieved as reflected in Fig 2. More importantly, using non-toxic reducing agent will not generate any toxic residual after the synthesis. Even though the photoactive GA based products were generally a black body materials, i.e. blockage of the visible light was taken place in the bulk products, such properties will not affect their optical properties when the optimum amount of the photoactive species like photosensitive metal oxides, or photosensitive II-VI metal sulphides was loaded to the GO throughout the photoactive GA synthesis from other reports.[1, 3] The possible reason was probably due to the effective charge transfer from the loaded materials in the 3D GA array which inhabited the electron-hole $\left(\mathrm{e}^{-} / \mathrm{h}^{+}\right)$pair recombination based on the works from other groups.[1, 3] The above-mentioned results of the present products had similar finding, which showed advanced point of current visible-light sensitive GA products via cleaner and user-friendly process with good photoactivity.

\subsection{Photocatalytic activity investigation in MO decomposition}


The presence of the absorption edge at $588 \mathrm{~nm}$ in CMPGA1 and $628.5 \mathrm{~nm}$ in CMPGA2 samples as shown in Fig 7 arouse our interest to observe the activity of the composite in the dye decomposition under visible-light irradiation. Fresh CMPGA samples were used for the MO degradation in light reaction after pre-adsorption equilibrium in the first part of the study. The result of the sequential dark/light reaction for $3 \mathrm{~h}$ as shown in Fig 8 reflected that the change in the normalized $\mathrm{MO}$ concentration $\left(\mathrm{C} / \mathrm{C}_{0}\right)$ throughout the period. In the dark period (0-100 min), the degree of $\mathrm{C} / \mathrm{C}_{0}$ variation was reduced when the BTZ-Py content in the GA increased from 2 $\mathrm{wt} \%$ to $5 \mathrm{wt} \%$, with the value of reduction of $65.4 \%$ in CMPGA1 and $35.5 \%$ in CMPGA2 for 10 mg of catalyst (Fig 8a). Once the visible light source was switched on, the MO concentration was reduced sharply, where the change of exact $\mathrm{C} / \mathrm{C}_{0}$ value was 0.3 in case of CMPGA1 (0.35 to 0.05$)$ and 0.33 for CMPGA2 (0.65 to 0.32 ). It primarily showed that the activity of CMPGA1 was close to that of CMPGA2 based on the $\mathrm{MO} \mathrm{C} / \mathrm{C}_{0}$ variation in light on period. In contrast, the variation of $\mathrm{MO}$ concentration during the light on period was very low (0.18 to 0.04$)$ after the dramatic reduction in the $\mathrm{MO}$ concentration during the dark period ( 1 to 0.18 ) when $10 \mathrm{mg}$ pure GA was used in the system. This showed that pure GA only acts as the adsorbent in the MO degradation and showed almost zero photocatalytic activity. At the same time, when pure BTZPy was used, the MO degradation was $48 \%$ (52\% reduction) in the dark period, and then further reduced to $27 \%$ of the initial concentration (from 0.52 to 0.27 ) in the light period, which was laid between that of CMPGA1 and CMPGA2. In the absence of the GA or BTZ-Py containing products, the MO concentration was almost stable (reduction of MO concentration was $8.2 \%$ ) since the self-degradation of MO was difficult in the sequential dark/light reaction. By comparing the MO concentration variation in the light on period for these three photochemically active materials (i.e. BTZ-Py, CMPGA1 and CMPGA2), it was found that their degree of MO 
degradation was similar, which may be due to the quick saturation of the MO for both the pure polymer and the polymer loaded GA array at low dosage level $(10 \mathrm{mg})$. When the amount of the GA based materials and the pure polymer was increased to $20 \mathrm{mg}$ (Fig $8 \mathrm{~b}$ ), the speed of MO removal in both dark and light reaction increased. Among them, the CMPGA1 have the highest rate in the dark adsorption of MO among the CMPGA group (83.4\% reduction), and consequently the lowest rate in the MO removal under visible-light turn on period due to the low initial MO concentration after the 100 min dark reaction. This is reflected from the very small $\mathrm{C} / \mathrm{C}_{0}$ variation (from 0.166 to 0.007 ) in the light reaction period for this material, which was slightly higher than that achieved by the pure GA (from 0.058 to 0.004 ). In contrast, the CMPGA2 had relatively weak activity in a dark reaction period with the small $\mathrm{C} / \mathrm{C}_{0}$ reduction by $49.6 \%$, while the activity was enhanced sharply in the light period with larger drop in the $\mathrm{C} / \mathrm{C}_{0}$ value from 0.504 to 0.084 . It was much stronger than the activity of the CMPGA1 (from 0.166 to 0.007), and slightly stronger than that of the pure BTZ-Py (from 0.48 to 0.19 ) in the same reaction. While in the case of the $20 \mathrm{mg}$ pure GA control, the degree of MO removal was very sharp in the dark reaction period $\left(94.2 \% \mathrm{C} / \mathrm{C}_{0}\right.$ reduction), followed by relatively small variation of the MO C/C $\mathrm{C}_{0}$ value in the light reaction period (from 0.058 to 0.004 ) as shown in Fig $8 \mathrm{~b}$. The tendency of mass variation for the same group of CMPGA used towards reactivity in the same study as shown in Fig 9 reflected that, the larger the mass of CMPGA used, the higher the speed of adsorption in the dark period as well as higher visible-light driven MO removal reaction rate. Based on the results from the series of studies mentioned above, it primarily showed excellent photocatalytic activity for the CMPGA2 in MO degradation among all CMPGA samples.

Although CMPGA showed good photocatalytic reaction tendency in MO degradation from previous part, since GA based materials were also a good adsorbent for different pollutants in 
many studies,[25-28] which may mask the actual photocatalytic activity of MO degradation exhibited by CMPGA under visible-light irradiation. So, the control investigation on the adsorption characteristics of the GA based samples need to be studied consequently. $20 \mathrm{mg}$ fresh freeze-dried pure GA or CMPGAs were mixed with $10 \mathrm{ppm}$ MO solution and the reaction was carried out under full dark and light environment for $3 \mathrm{~h}$ without pre-equilibrium step in order to study the effect of the adsorption towards the photocatalytic reactions in this part. The result illustrated Fig 10 in showed that the activity of CMPGA2 in dark environment was weaker than that in the visible-light environment base on the change in the normalized MO concentration $\left(\mathrm{C} / \mathrm{C}_{0}\right)$, which is reflected from the results obtained in Table 2 where the adsorption efficiency in dark environment $(50.0 \%)$ was lower than that in light on reaction $(90.4 \%)$, that means activity of the dark adsorption was $55 \%$ of the overall reaction. It showed that under the light on environment, adsorption reaction contributed half of the reaction. The adsorption ability was also not strong based on the results recorded in the dark reaction. In contrast, the dark adsorption efficiency achieved by CMPGA1 $(83 \%)$ was slightly smaller than that in the light on reaction (89.1\%). The tendency of the dark reaction activity achieved by the pure GA and CMPGAs in this part was similar to that recorded in Figs 8-9 before the light source turned on, where the polymer in loaded GA affected the adsorption ability. This primarily showed that when the amount of the photosensitive polymer loaded in the GA array increased, the space available for the adsorption gradually reduced. While the photocatalytic activity of the GA becomes dominating due to increasing content of the photochemically sensitive polymer in the GA as demonstrated in their DRS spectra (Fig 7). Such phenomena were further confirmed from the fresh pure GA control sample under the identical environment as the activity of pure GA was similar in the fully dark (86.6\% removal) and fully light on $(86.2 \%$ removal) environment, and 
similar in the $\mathrm{C} / \mathrm{C}_{0}$ variation tendency. General speaking, the over tendency of the $\mathrm{GA}$ and CMPGAs in the photocatalytic reaction (Figs 8-9) and the full dark and full light environment (Fig 10) showed that the BTZ-Py loaded in the GA played an important role in the photocatalytic MO degradation, and the activity increased up on the increase in the BTZ-Py loading in the CMPGA.

The generalized results mentioned above (Figs 8 and 10) were similar to those reactions which involved the use of other GA or graphene based photocatalysts based on the $\mathrm{C} / \mathrm{C}_{0}$ trends against time.[1,3] This also reflected that the pure GA totally involved adsorption only. The MO degradation by the CMPGA and pure BTZ-Py were mainly achieved by the physical adsorption during the dark period, but photocatalytic reaction was the dominated one during light-on period since the light was switched on after the saturation of the photocatalyst. By comparing the activity of the GA based materials in the MO decomposition by visible-light as listed in Tables 2-4, it showed that activity of the 20 mg CMPGA2 (91.6\%) were comparable to other reported materials like $\mathrm{BiOBr} / \mathrm{RGO}$ aerogel $(94.7 \%)$ and CNGA (94\%) under similar operation. The only difference is that the time of the photocatalytic MO degradation achieved in the current study $(80 \mathrm{~min})$ is shorter than other GA based photocatalysts $(2-4 \mathrm{~h})$ and lower mass is required.[1, 3] It primarily showed that the CMPGA had stronger photocatalytic activity in the visible-light driven MO decomposition after the saturated adsorption process.

Since the initial MO concentration for the light on period for different photocatalysts (i.e. CMPGA, BTZ-Py, or pure GA) were different, and reported works on the GA based photocatalytic MO decomposition also quantified the photocatalytic activity based on the final $\mathrm{C} / \mathrm{C}_{0}$ value recorded in the end of the reaction. $[1,3,8,14,15]$ More importantly, the starting concentration of the dye after the dark adsorption equilibrium process in these works was 
different in the light on reactions which were similar to the practice in first part of our current study. $[1,3,8,14,15]$ As a result, it is unfair for activity comparison as reflected from Figs 8 and 9, and the actual photocatalytic activity cannot be fully reflected from reaction results obtained based on the operation from the reported works.[1, 3, 8, 14, 15] An extra investigation of MO removal for the light on period was carried out by firstly saturating the MO solution under dark condition for 2 hours. Then, the residual solution was removed and the MO saturated pure GA, BTZ-Py, and CMPGA samples with mass of 10 and $20 \mathrm{mg}$ were extracted and put into a fresh 10 ppm MO under the light on with the same time period as in previous experiment (i.e. $80 \mathrm{~min}$ ). The results recorded in Fig 11 showed the photocatalytic activity and corresponding kinetics of the MO saturated BTZ-Py, CMPGA, and pure GA grouped with the same mass during the 80 min visible-light driven MO degradation reaction. When the mass of the catalyst was set at 10 mg (Fig 11a), the activity of the CMPGAs and pure BTZ-Py was very similar with each other. Even though the CMPGA2 achieved the strongest MO removal activity with the reduction in $\mathrm{C} / \mathrm{C}_{0}$ value by $70.7 \%$, the reduction in $\mathrm{C} / \mathrm{C}_{0}$ recorded for the CMPGA1 and pure the BTZ-Py were $68.9 \%$ and $69.3 \%$, respectively. No significant difference in activity was found between them. At the same time, pure GA achieved the worst activity as only $5.9 \%$ of MO was decomposed. In contrast, the difference in the activity was clear when the amount of the materials used was increased to $20 \mathrm{mg}$ (Fig 11b). The CMPGA1 achieved the lowest activity among the CMPGA samples in the visible-light assisted MO removal reaction with the reduction of $\mathrm{C} / \mathrm{C}_{0}$ value by $51.2 \%$ throughout 80 min reaction. On the other hand, even though the $\mathrm{C} / \mathrm{C}_{0}$ reduction achieved by pure the BTZ-Py was $86.9 \%$, it was still smaller than that achieved by the CMPGA2 (89.2\%). In contrast, pure GA only achieved 6.5\% MO concentration reduction after the saturation step, which showed almost no photocatalytic activity in MO degradation. 
Additional study of the simulated kinetic analysis of the MO saturated CMPGA reactions based on Figs 11a-11b in terms of absolute MO concentration was carried out. Results in Fig 11c-11d showed that they had good fitting with the first order reaction kinetics, which was similar to tendency demonstrated by Tong's group.[1] In-depth analysis showed that when the catalyst was $10 \mathrm{mg}$ (Fig 11c), the kinetics was slow and difference was small, even though CMPGA2 still can achieve the highest rate of reaction $\left(0.0149 \mathrm{~min}^{-1}\right)$ when compared to that of CMPGA1 (0.0139 $\left.\min ^{-1}\right)$ and BTZ-Py alone $\left(0.0144 \mathrm{~min}^{-1}\right)$. However, kinetics of reaction was increased with obvious difference when the catalyst amount increased to $20 \mathrm{mg}$. It reflected from the rate strongest obtained in CMPGA2's system $\left(0.0269 \mathrm{~min}^{-1}\right)$ compared with those of CMPGA1 $\left(0.0087 \mathrm{~min}^{-1}\right)$ and BTZ-Py $\left(0.0249 \mathrm{~min}^{-1}\right)$. The tendency of the $\mathrm{C} / \mathrm{C}_{0}$ variation in the light on period in this experiment highly matched with those obtained in Fig 10, which proved that the CMPGA really exhibited photochemical activity under light on period instead of the adsorption of chemical, even though the catalyst was saturated with the MO under the dark period, i.e. dark adsorption equilibrium achieved before the removal of residual or refreshing MO in the solution.

Generalizing the photocatalytic degradation results obtained under different reaction parameters with control works as illustrated from Figs 8-11, they showed that the activity of the CMPGA with higher loading in the GA array had better performance. The difference in activity was especially obvious after $20 \mathrm{mg}$ of CMPGAs, BTZ-Py and pure GA saturated with MO overnight (i.e. adsorption equilibrium achieved) before the reaction in fresh MO solution. Pure GA showed no photocatalytic activity, while photocatalytic activity of materials in ascending order of CMPGA1<BTZ-Py $<$ CMPGA2. Such phenomenon was especially obvious at the case of $20 \mathrm{mg}$ system. Generally speaking, enhancement of activity resulted from the synergistic effect between the BTZ-Py and GA array throughout the CMPGA synthesis possibly in two ways, (i) 
the change of the chemical structure in the hybridization of BTZ-Py with GO under mild hydrothermal reaction; (ii) and the low BTZ-Py equivalent amount in the CMPGA achieving slightly better activity than pure BTZ-Py under identical mass $(20 \mathrm{mg})$ of bulk materials. The possible reason for the first hypothesis may be arisen from the DRS absorption edge (Fig 7) contributed from the BTZ-Py in the CMPGA was enhanced upon the increase in the BTZ-Py loading. Since increase in the amount of BTZ-Py in the BTZ-Py/GO raw solution in the CMPGA synthesis resulted in higher loading percentage of BTZ-Py in CMPGA product (Table 1), and hence the red shifting of the absorption edge from $588 \mathrm{~nm}$ in CMPGA1 to $628.5 \mathrm{~nm}$ in CMPGA2. Such phenomenon was especially important for the absorption of visible-light. In addition to the zero wt $\%$ of S in EDX result of pure GA (Table 1), the existence of C-S-C bonding peak in S2p HR-XPS (Figs 6g and 6j), and N1s HR-XPS of CMPGAs (Figs 6f and 6i) shared similar peak pattern $\left(\mathrm{N}_{\mathrm{Q}}\right.$ : $399.7-399.8 \mathrm{eV}$ and graphitic $\left.\mathrm{N}: 402.1-402.5 \mathrm{eV}\right)$ with that of BTZ-Py $(401.8 \mathrm{eV}$, Fig 6o). These showed there was chemical interaction between GO and BTZ-Py in the mild conditioned hydrothermal reduction, and existence of $\pi-\pi$ interaction between the graphene skeleton and the loaded BTZ-Py in CMPGA. All these results showed the change in electronic structure of the BTZ-Py was taken place in certain extent upon the CMPGA synthesis. Such effect enhances the photoelectron transfer to the MO solution from CMPGA2 without recombination of electron-hole $\left(\mathrm{e}^{-} / \mathrm{h}^{+}\right)$pair based on the DRS spectrum shown in Fig 7, and hence the enhancement of CMPGA's photocatalytic activity. Findings mentioned above finally caused the enhancement in the photocatalytic degradation of MO by the CMPGA2. In general cases, the black body properties of the photoactive GA materials with the optimum amount of the photoactive species will not affect their photochemical activity when they were applied in the photochemical reactions. Such as photosensitive metal oxides or $\mathrm{g}-\mathrm{C}_{3} \mathrm{~N}_{4}$ based materials was 
loaded into the GA array throughout the synthesis.[1-3, 8, 14, 15] The possible reason may be due to the effective transfer of photoelectrons generated from the loaded materials to the large GO nanosheets surface freely in the GA array. This is important for the inhibition of the recombination of the $\mathrm{e}^{-} / \mathrm{h}^{+}$pair, which enhanced the photocatalytic activity in the visible-light driven reaction and results in the strong UV-vis peak of the composite.[1-3] Based on the results obtained in Fig 7, similar trend was observed after the loading of BTZ-Py to the GO throughout the aerogel formation, and the peak intensity increased upon the increase in the polymer content. This may be a possible reason on the enhancement of the photocatalytic activity in the visible light driven MO photodecomposition recorded in Figs 8-11, especially CMPGA2.

In the hypothesis of synergistic effect by the low equivalent amount of loaded BTZ-Py for CMPGA activity enhancement, since pure BTZ-Py exhibited strong activity and kinetics of reaction (Fig 11) by its DRS absorption edge at visible light region (Fig 7a), which made little difference in activity between the pure BTZ-Py and the CMPGA2. It was similar to some of the GA based materials catalyzed visible-light driven photocatalytic decomposition.[4, 12, 18] However, the strong activity of MO photocatalytic decomposition achieved by $20 \mathrm{mg}$ neat BTZPy (Fig $11 \mathrm{~b}, 86.9 \%$ ) was totally contributed from the polymer itself (i.e. $100 \mathrm{wt} \%$ polymer). Relative to the low activity recorded in CMPGA1 (51.2\%, Fig $11 \mathrm{~b})$, activity of CMPGA2 was elevated to $89.2 \%$ (Fig $11 \mathrm{~b}$ ). Even though the difference of apparent $\mathrm{C} / \mathrm{C}_{0}$ achieved between neat polymer and CMPGA2 was small with drop of activity in CMPGA1, the wt $\%$ of BTZ-Py in the CMPGA1 and CMPGA2 was only $2 \%$ and $5 \%$ (i.e. $0.4 \mathrm{mg}$ and $1 \mathrm{mg}$ equivalent of BTZ-Py), respectively. In other words, since photocatalytic ingredient was contributed from BTZ-Py (Fig 7), strong activity of MO degradation achieved by neat BTZ-Py required $20 \mathrm{mg}$ of polymer (i.e. $100 \mathrm{wt} \%$ ) from Fig 11c, but similar activity achieved by CMPGA2 (5 wt\% BTZ-Py) only 
required $1 \mathrm{mg}$ equivalent of BTZ-Py loaded inside GA array. The weak activity achieved by CMPGA1 (51.2\%) was possibly caused by even lower amount of BTZ-Py (2 wt\%, $0.4 \mathrm{mg}$ equivalent) loaded in the GA array of CMPGA1. Combining the XPS, DRS findings mentioned above, these phenomena finally caused the enhancement in the photocatalytic degradation of MO by the CMPGA2.

On the other hand, GA based materials were good adsorbent[25-28] which may have risk of competition between adsorption and photocatalytic activity as mentioned previously. Up to date, most of the GA based photocatalytic investigation were carried out after the dark adsorption equilibrium achieved immediately, $[1,3,5,8,9,12-15,18]$ which was similar to results obtain in Figs 8-9 in current study. The unequal initial dye concentration in the light turn on period was also counted in study. Even though some of the previous work investigated activity of the fresh GA based photocatalyst under full dark and full light (UV and vis-light) condition,[2] which was similar to results obtained in Fig 10 of current work. However, the activity difference was very not obvious.[2] More importantly, their overall activity was accounted in terms of the final $\mathrm{C} / \mathrm{C}_{0}$ value. As the result, risk of actual activity of the photocatalyst being masked by its adsorption ability was then exist. With the help of systematic control experiments via the use of as-prepared GA based materials (GA and CMPGAs) under full dark and full light on environment (Fig 10), and the use of MO saturated photocatalysts (CMPGAs, GA, and BTZ-Py) after achieving overnight dark equilibrium for the full light degradation of $\mathrm{MO}$ with identical initial concentration (Fig 11) as described previously. It totally showed that when BTZ-Py loading in CMPGA increased, photocatalyst character becomes dominant character gradually. Even though BTZ-Py itself was nanopolymer in nature, it was in the form of powder instead of bulk block shape of nanoporous GA products. As a result, the activity of the adsorption was limited based 
on results shown in Fig 8. In contrast, pure GA acted as an adsorbent only as demonstrated by its very weak photocatalytic activity after the MO saturation $(<7 \%)$. This reflected from the results obtained in Fig 11 and similar performance of the rapid MO concentration reduction achieved in either dark or light on environment, especially for the $20 \mathrm{mg}$ cases (Figs $8 \mathrm{~b}$ and 10). It was possibly due to the enormous active surface area for the MO molecules' adsorption in the GNs array of the GA sample, which reflected from the difference in the sample morphology reflected from the TEM images of the CMPGA, pure GA and BTZ-Py as illustrated in Fig 5. As a result, pure GA showed zero activity in the photocatalytic degradation of MO, while CMPGA2 actually exhibited photocatalytic activity as shown in Fig 11.

Summarizing the previous explanation on the synergistic effect based on the change of electronic structure via XPS and DRS, and achievement of strong photocatalytic activity by relatively small equivalent amount (1 mg equivalent) of photoactive BTZ-Py in CMPGA compared to pure BTZ-Py under identical bulk sample mass $(20 \mathrm{mg})$. It showed that CMPGA with the polymer loading of $5 \mathrm{wt} \%$ had the strongest overall photocatalytic activity for the MO removal under visible-light.

The reusability of the CMPGA was also the major concern since it affected the practical value of the CMPGA in the waste reduction, a 3 cycles operations from the fresh sample was carried out in the present study similar to those carried out by other groups in the photodegradation of MO using GA based visible-light driven photocatalysts (3-5 cycles).[1, 3, 8, 14, 15] Results shown in Fig 12 revealed that the reduction of $\mathrm{C} / \mathrm{C}_{0}$ at the end of the 3 operation cycles was $91.8 \%, 88.1 \%$ and $87.4 \%$, respectively, a slight drop of $4.4 \%$ after the three cycles. The tendency of the activity variation (Fig 12) was similar to those recorded by other groups on the MO photodecomposition.[1, 3, 8, 14, 15] Since the photocatalyst was recycled by centrifuge 
extraction from the used MO solution, such results reflected that the CMPGA catalyst was chemically stable upon magnetic stirring process with low activity depreciation upon recycling. By comparing the activity of the GA based materials in the MO decomposition by visible-light as listed in Tables 2-4 and Fig 12. It showed that the CMPGA had excellent photocatalytic activity under in the visible-light driven MO decomposition after the adsorption equilibrium. Such strength made it became an excellent candidate of visible-light driven metal-free photocatalyst.

\section{Conclusions}

The photosensitive GA was successfully produced from a green and mild conditioned hydrothermal reaction of GO and BTZ-Py. The GA produced exhibited $\pi-\pi$ interaction between the reduced graphene stacks and the polymer molecules based on the EDX mapping pattern and XPS results. It also exhibited strong optical properties at the visible range from the UV-visible DRS spectroscopy. The CMPGA with BTZ-Py loading of $5 \mathrm{wt} \%$ showed strong photocatalytic activity in the MO decomposition under a visible light source with a percentage of removal greater than $90 \%$, which outperformed to the other GA based photocatalyst in the same reaction. The strong activity achieved by the $20 \mathrm{mg}$ MO saturated CMPGA2 (5 wt\% BTZ-Py) $(89.2 \%)$ in the visible light driven MO photodecomposition was comparable to that of $20 \mathrm{mg}(100 \mathrm{wt} \%)$ MO saturated BTZ-Py (86.9\%). It was possibly due to the synergistic effect between GA and BTZ-Py by (i) change in chemical structure after hybridizing BTZ-Py with GO throughout CMPGA formation; and (ii) the enhancement of the photocatalytic activity exhibited by small BTZ-Py equivalent amount ( $1 \mathrm{mg}$ equivalent) in the CMPGA achieving compared with pure BTZ-Py and pure GA under identical mass $(20 \mathrm{mg})$ of bulk materials. The stable activity 
throughout the 3 cycles operations showed that CMPGA was an excellent metal-free photocatalyst. Its potential applications in other areas with investigations are expected in future.

\section{Acknowledgement}

This work described in this paper was substantially supported by a grant from the Hong Kong-Scotland Partners in Post Doctoral Research Scheme Supported from the Research Grants Council of Hong Kong and the Scotland Government (S-HKU702/15), Natural Science Foundation of China (NSFC) and the Research Grants Council (RGC) of Hong Kong Joint Research Scheme (No. 51561165015, No.N_HKU718/15), and the UK Engineering and Physical Sciences Research Council (EPSRC) under grant number EP/R012164/1. The XPS results were contributed from the IAM centre of Hong Kong Baptist University (HKBU) and Suzhou Deyo Bot Advanced Materials Co., Ltd.

\section{References:}

[1] Tong ZW, Yang D, Shi JF, Nan YH, Sun YY, Jiang ZY. Three-Dimensional Porous Aerogel Constructed by g- $_{3} \mathrm{~N}_{4}$ and Graphene Oxide Nanosheets with Excellent Visible-Light Photocatalytic Performance. ACS Appl Mater Interfaces. 2015;7:25693-701.

[2] Hou C, Zhang Q, Li Y, Wang H. P25-graphene hydrogels: Room-temperature synthesis and application for removal of methylene blue from aqueous solution. J Hazard Mater. 2012;205206:229-35

[3] Yu X, Shi JJ, Feng LJ, Li CH, Wang L. A three-dimensional BiOBr/RGO heterostructural aerogel with enhanced and selective photocatalytic properties under visible light. Appl Surf Sci. 2017;396:1775-82.

[4] Yang MQ, Zhang N, Wang Y, Xu YJ. Metal-free, robust, and regenerable 3D grapheneorganics aerogel with high and stable photosensitization efficiency. J Catal. 2017;346:21-9.

[5] Wan WC, Yu S, Dong F, Zhang Q, Zhou Y. Efficient $\mathrm{C}_{3} \mathrm{~N}_{4} /$ graphene oxide macroscopic aerogel visible-light photocatalyst. J Mater Chem A. 2016;4:7823-9.

[6] Vilela F, Zhang K, Antonietti M. Conjugated porous polymers for energy applications. Energy Environ. Sci. 2012;5:7819-32.

[7] Zhang K, Kopetzki D, Seeberger PH, Antonietti M, Vilela F. Surface Area Control and Photocatalytic Activity of Conjugated Microporous Poly(benzothiadiazole) Networks. Angew Chem-Int Edit. 2013;52:1432-6.

[8] Cai JY, Liu WJ, Li ZH. One-pot self-assembly of $\mathrm{Cu}_{2} \mathrm{O} / \mathrm{RGO}$ composite aerogel for aqueous photocatalysis. Appl Surf Sci. 2015;358:146-51. 
[9] Ma YH, Wang JJ, Xu SM, Feng S, Wang JD. $\operatorname{Ag}_{2} \mathrm{O}$ /sodium alginate-reduced graphene oxide aerogel beads for efficient visible light driven photocatalysis. Appl Surf Sci. 2018;430:155-64.

[10] Zhang X, Sui Z, Xu B, Yue S, Luo Y, Zhan W, et al. Mechanically strong and highly conductive graphene aerogel and its use as electrodes for electrochemical power sources. $\mathrm{J}$. Mater. Chem. 2011;21:6494-7.

[11] Hu H, Zhao Z, Wan W, Gogotsi Y, Qiu J. Ultralight and Highly Compressible Graphene Aerogels. Adv Mater. 2013;25:2219-23.

[12] Fan CY, Liu QQ, Ma TD, Shen JY, Yang Y, Tang H, et al. Fabrication of 3D $\mathrm{CeVO}_{4} /$ graphene aerogels with efficient visible-light photocatalytic activity. Ceram. Int. 2016;42:10487-92.

[13] Nawaz M, Miran W, Jang J, Lee DS. One-step hydrothermal synthesis of porous 3D reduced graphene oxide/ $/ \mathrm{TiO}_{2}$ aerogel for carbamazepine photodegradation in aqueous solution. Appl Catal B-Environ. 2017;203:85-95.

[14] Tang L, Jia CT, Xue YC, Li L, Wang AQ, Xu G, et al. Fabrication of compressible and recyclable macroscopic $\mathrm{g}_{-} \mathrm{C}_{3} \mathrm{~N}_{4} / \mathrm{GO}$ aerogel hybrids for visible-light harvesting: A promising strategy for water remediation. Appl Catal B-Environ. 2017;219:241-8.

[15] Yan XR, Xu RP, Guo JK, Cai X, Chen DJ, Huang LH, et al. Enhanced photocatalytic activity of $\mathrm{Cu}_{2} \mathrm{O} / \mathrm{g}-\mathrm{C}_{3} \mathrm{~N}_{4}$ heterojunction coupled with reduced graphene oxide three-dimensional aerogel photocatalysis. Mater Res Bull. 2017;96:18-27.

[16] Jiang JX, Su F, Trewin A, Wood CD, Campbell NL, Niu H, et al. Conjugated microporous poly (aryleneethynylene) networks. Angew Chem-Int Edit. 2007;46:8574-8.

[17] Wong YL, Tobin JM, Xu Z, Vilela F. Conjugated porous polymers for photocatalytic applications. J Mater Chem A. 2016;4:18677-86.

[18] Yu X, Wu PW, Qi CX, Shi JJ, Feng LJ, Li CH, et al. Ternary-component reduced graphene oxide aerogel constructed by $\mathrm{g}-\mathrm{C}_{3} \mathrm{~N}_{4} / \mathrm{BiOBr}$ heterojunction and graphene oxide with enhanced photocatalytic performance. J. Alloys Comp. 2017;729:162-70.

[19] Kovtyukhova NI, Ollivier PJ, Martin BR, Mallouk TE, Chizhik SA, Buzaneva EV, et al. Layer-by-layer assembly of ultrathin composite films from micron-sized graphite oxide sheets and polycations. Chem Mat. 1999;11:771-8.

[20] Chen P, Yang J-J, Li S-S, Wang Z, Xiao T-Y, Qian Y-H, et al. Hydrothermal synthesis of macroscopic nitrogen-doped graphene hydrogels for ultrafast supercapacitor. Nano Energy. 2013;2:249-56.

[21] Yu X, Park SK, Yeon SH, Park HS. Three-dimensional, sulfur-incorporated graphene aerogels for the enhanced performances of pseudocapacitive electrodes. J Power Sources. 2015;278:484-9.

[22] Yu X, Kang Y, Park HS. Sulfur and phosphorus co-doping of hierarchically porous graphene aerogels for enhancing supercapacitor performance. Carbon. 2016;101:49-56.

[23] Balasubramanyan S, Sugunan S, Narayanan BN. Nitrogen-doped sulphonated 3dimensional holey graphene nanoarchitecture for selective oxidation of ethylbenzene. J Mater Sci. 2018;53:12079-90.

[24] Lu ZW, Chen YJ, Liu ZE, Li AQ, Sun D, Zhuo KL. Nitrogen and sulfur co-doped graphene aerogel for high performance supercapacitors. RSC Adv. 2018;8:18966-71.

[25] Moon IK, Yoon S, Chun K-Y, Oh J. Highly Elastic and Conductive N-Doped Monolithic Graphene Aerogels for Multifunctional Applications. Adv Funct Mater. 2015;25:6976-84. 
[26] Li J, Li J, Meng H, Xie S, Zhang B, Li L, et al. Ultra-light, compressible and fire-resistant graphene aerogel as a highly efficient and recyclable absorbent for organic liquids. J Mater Chem A. 2014;2:2934-41.

[27] He Y, Liu Y, Wu T, Ma J, Wang X, Gong Q, et al. An environmentally friendly method for the fabrication of reduced graphene oxide foam with a super oil absorption capacity. J Hazard Mater. 2013;260:796-805.

[28] Mi X, Huang G, Xie W, Wang W, Liu Y, Gao J. Preparation of graphene oxide aerogel and its adsorption for $\mathrm{Cu}^{2+}$ ions. Carbon. 2012;50:4856-64. 
Table captions:

Table 1. Elemental information of the $\mathrm{C}, \mathrm{O}$, and $\mathrm{S}$ composition in CMPGA samples vs pure GA based on EDX analysis (at $\%$ and $w t \%$ based).

Table 2. Activity of the CMPGAs vs pure GA $(20 \mathrm{mg})$ in the fully dark and full light on MO photodecomposition (3h).

Table 3. Activity of the representative GA based catalyst in the vis-light driven MO photodecomposition.

Table 4. Activity of the representative GA based catalyst in the vis-light driven MO photodecomposition at 60 th min after the light on reaction started.

Figure captions:

Fig 1. Theoretical repeat unit of the BTZ-Py.

Fig 2. Digital image of the bulk CMPGA1 (left) and CMPGA2 (right).

Fig 3. (a)-(d) SEM image of the CMPGA2 composite at different magnification (Scale bar: (a) $100 \mu \mathrm{m}$, (b) $50 \mu \mathrm{m}$, (c) $20 \mu \mathrm{m}$, (d) $10 \mu \mathrm{m}$ ), and (e) corresponding survey EDX spectrum; (f)-(i) SEM image and (j) EDX spectrum of pure GA; and (k)-(n) SEM image and (o) EDX spectrum of pure BTZ-Py. (Scale bar: (f, k) $100 \mu \mathrm{m},(\mathrm{g}, \mathrm{l}) 50 \mu \mathrm{m},(\mathrm{h}, \mathrm{m}) 20 \mu \mathrm{m}$, (i, n) $10 \mu \mathrm{m})$

Fig 4. EDX mapping of (a-b) $\mathrm{C}-\mathrm{K} \alpha$, (c-d) $\mathrm{O}-\mathrm{K} \alpha$, (e-f) $\mathrm{S}-\mathrm{K} \alpha$, (g-h) corresponding SEM image. of the CMPGA1 (Scale bar: $20 \mu \mathrm{m}$ ), CMPGA2 (Scale bar: $100 \mu \mathrm{m}$ ), (i) C-Ka, (j) O-Ka, (k) $\mathrm{S}-\mathrm{K} \alpha$, (l) corresponding original SEM image of pure GA, and (m) C-Ka, (n) O-Ka, (o) S$\mathrm{K} \alpha,(\mathrm{p})$ corresponding original SEM image of pure BTZ-Py. (Scale bar: (i-p) $50 \mu \mathrm{m}$ )

Fig 5. TEM of the (a)-(d) CMPGA2, (e-h) pure GA, and (i-1) pure BTZ-Py at different modifications. (Scale bar: (a) $0.2 \mu \mathrm{m}$, (b, f, j) $100 \mathrm{~nm}$, (c, g, h, k, l) $50 \mathrm{~nm}$, (d) $20 \mathrm{~nm}$, (e, i) $500 \mathrm{~nm})$

Fig 6. Survey XPS spectrum of the (a) CMPGA1, (b) CMPGA2, (c) pure GA and (d) pure BTZPy, and HR-XPS spectra (e) C1s, (f) N1s, and (g) S2p of CMPGA1; (h) C1s, (i) N1s, and (j) S2p of CMPGA2; (k) C1s, (l) N1s, and (m) S2p of pure GA; and (n) C1s, (o) N1s and (p) $\mathrm{S} 2 \mathrm{p}$ of pure BTZ-Py.

Fig 7. UV-visible spectrum (a) pure BTZ-Py, pure GA, and CMPGA with different content of BTZ-Py, and (b) zoom up of (a) on the CMPGA series.

Fig 8. The $\mathrm{C} / \mathrm{C}_{0}$ variation of MO throughout $3 \mathrm{~h}$ of the dark/light reaction of BTZ-Py, CMPGA with different polymer loading and pure GA (a) $10 \mathrm{mg}$, (b) $20 \mathrm{mg}$.

Fig 9. The $\mathrm{C} / \mathrm{C}_{0}$ variation of $\mathrm{MO}$ throughout $3 \mathrm{~h}$ of the dark/light reaction in presence of CMPGAs with different mass (a) $10 \mathrm{mg}$, (b) $20 \mathrm{mg}$.

Fig 10. (a) The activity of fresh pure GA, CMPGA1, and CMPGA2 $(20 \mathrm{mg})$ in the MO removal under fully dark and fully visible-light illumination environment (3h).

Fig 11. The $\mathrm{C} / \mathrm{C}_{0}$ variation of MO throughout $80 \mathrm{~min}$ of the light reaction in presence of BTZ-Py, CMPGA, and pure GA with different polymer loading after saturated with MO (a) $10 \mathrm{mg}$, (b) $20 \mathrm{mg}$, and the corresponding chemical kinetics fitting plots (c) $10 \mathrm{mg}$, and (d) $20 \mathrm{mg}$.

Fig 12. Recyclability of the CMPGA2 for photodegradation of MO (3 cycles). 
Figures

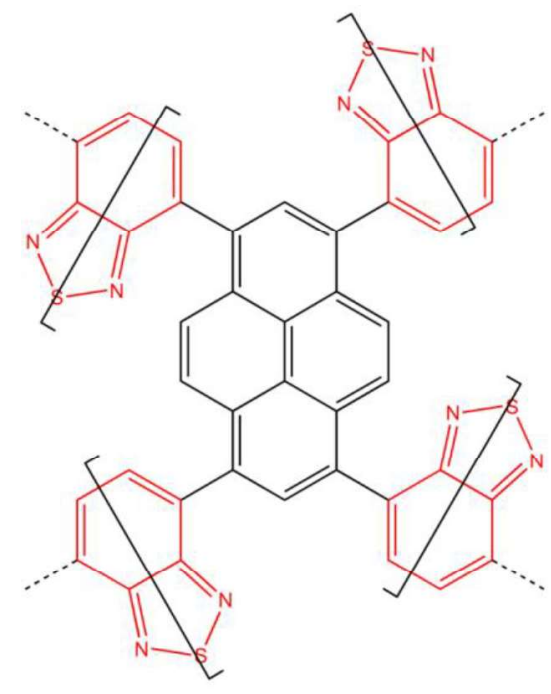

BTZ-Py polymer

Fig 1 


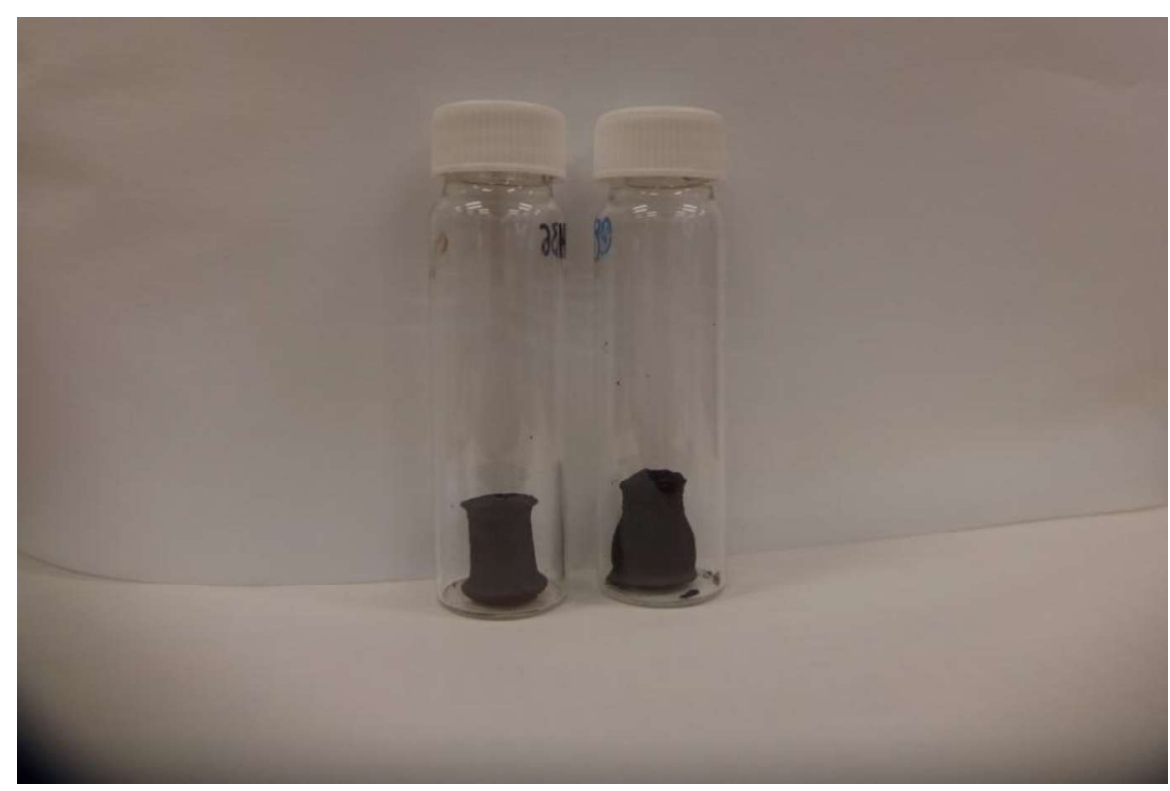

Fig 2 


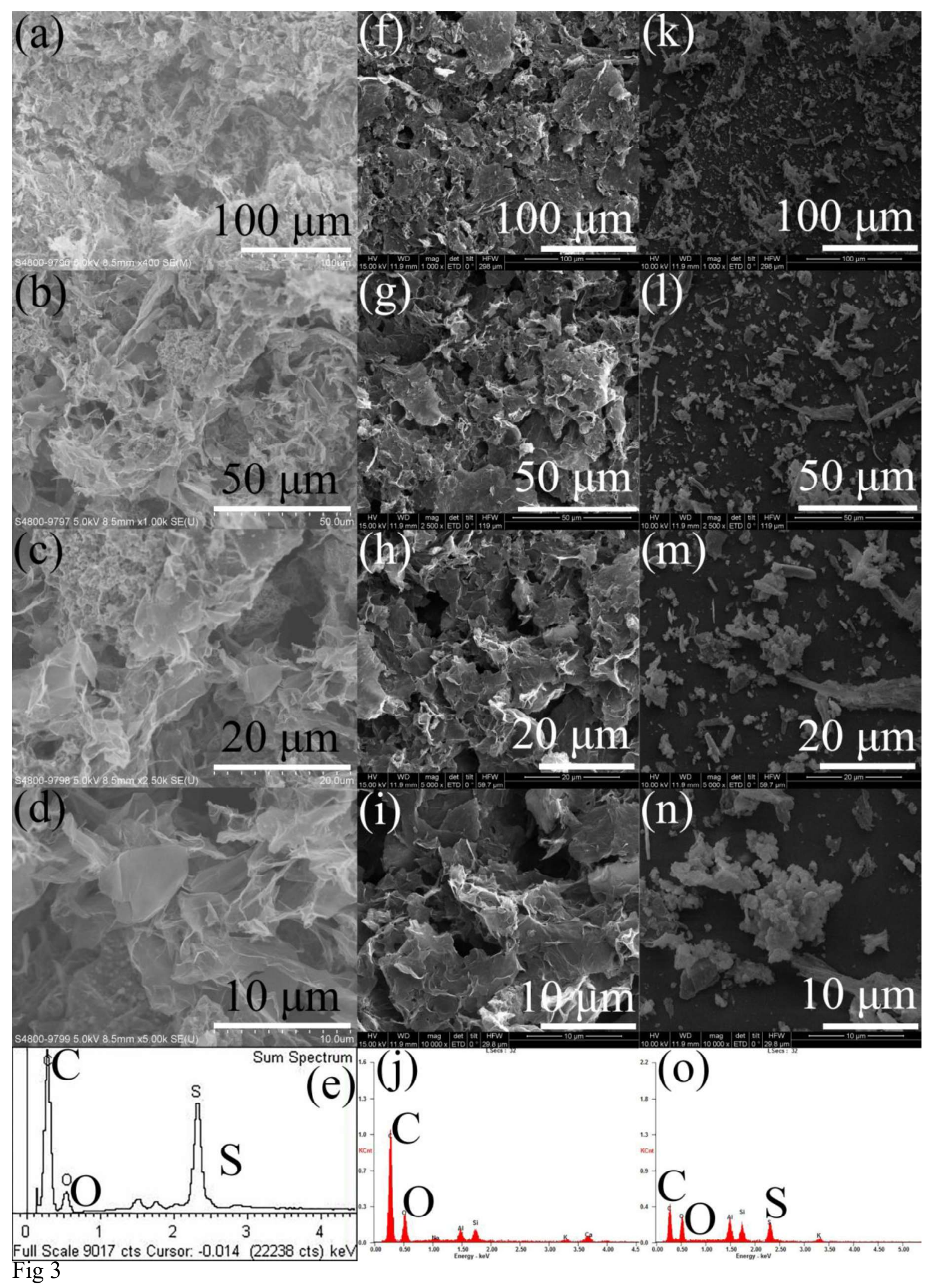




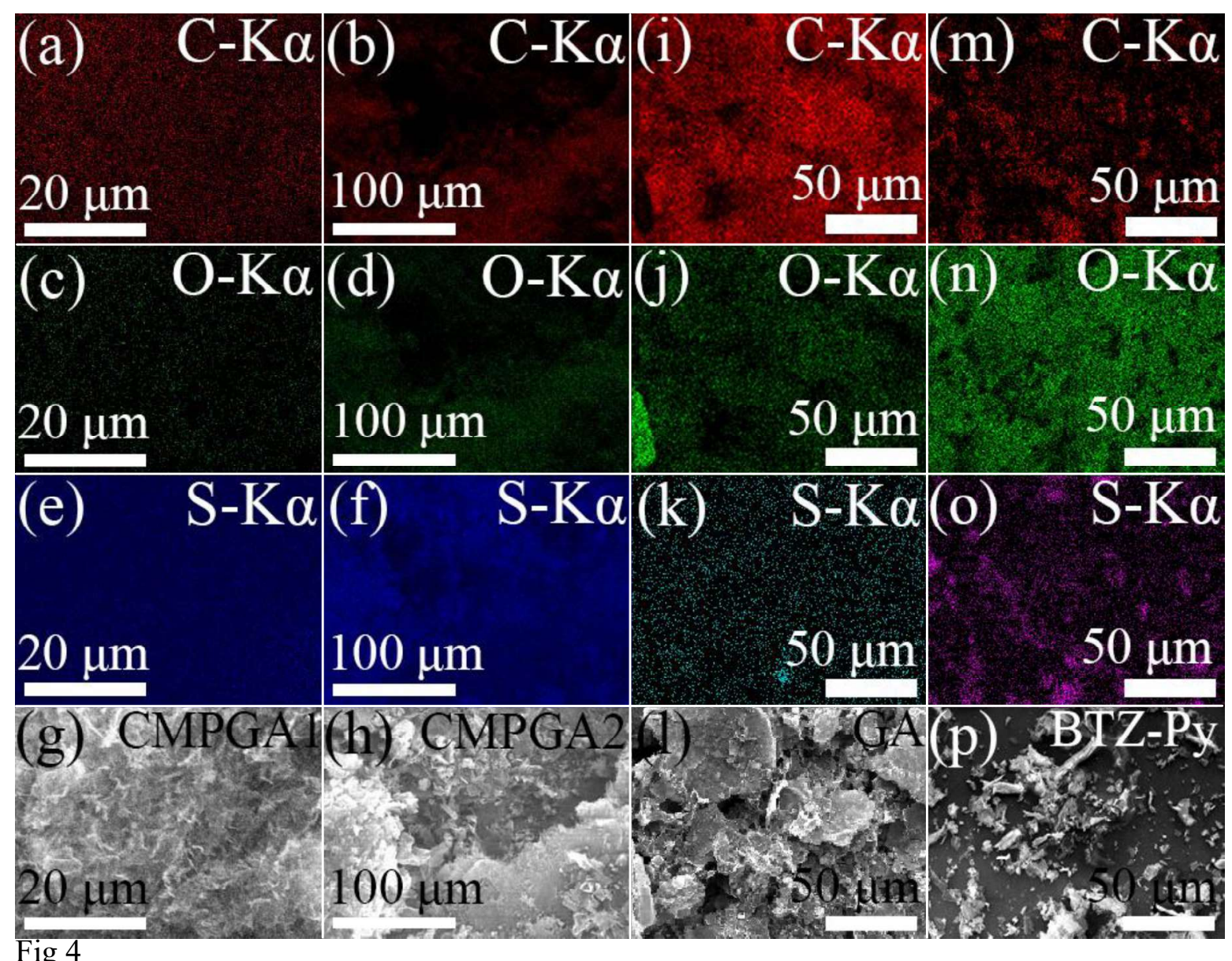




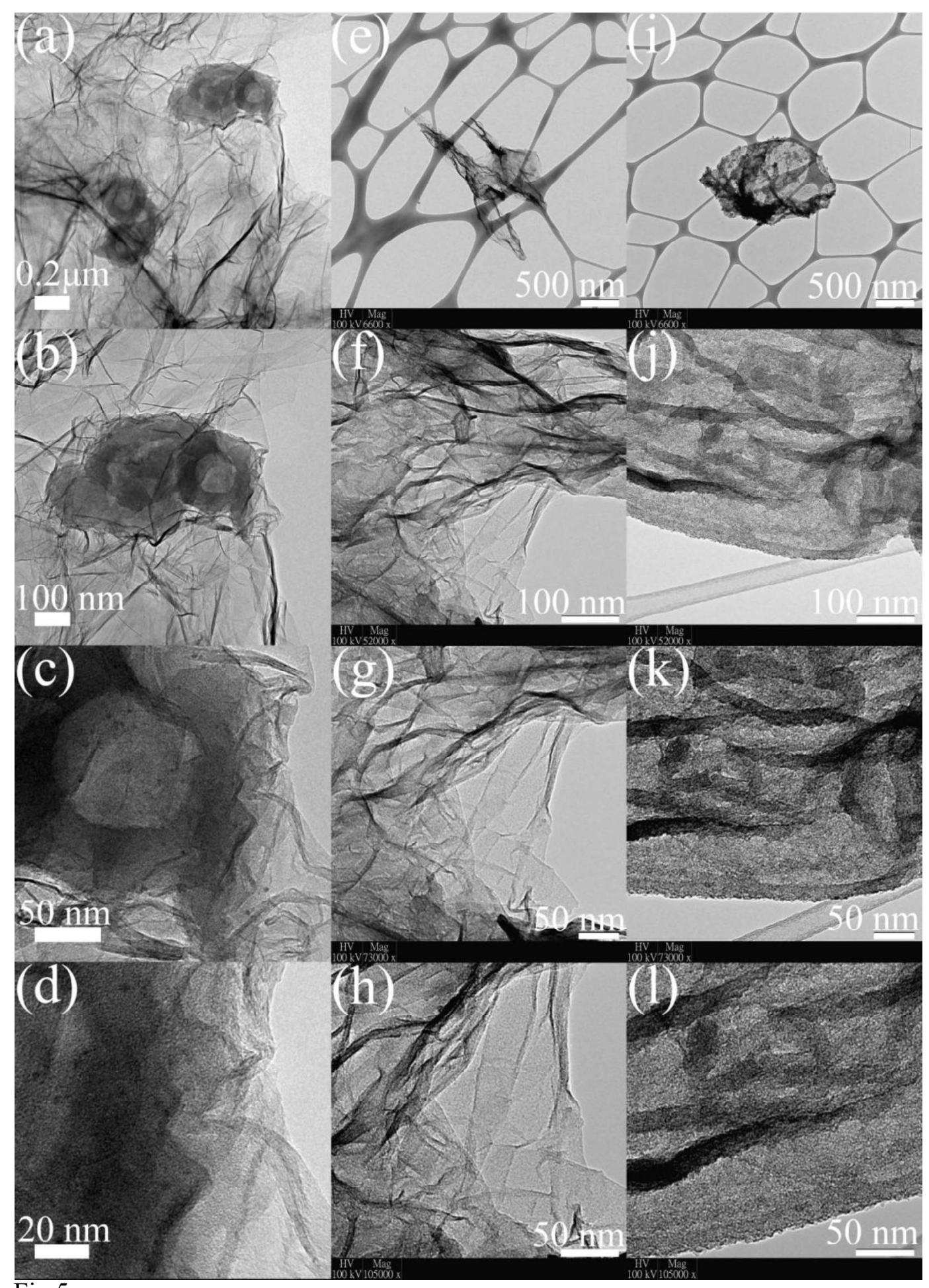

Fig 5 

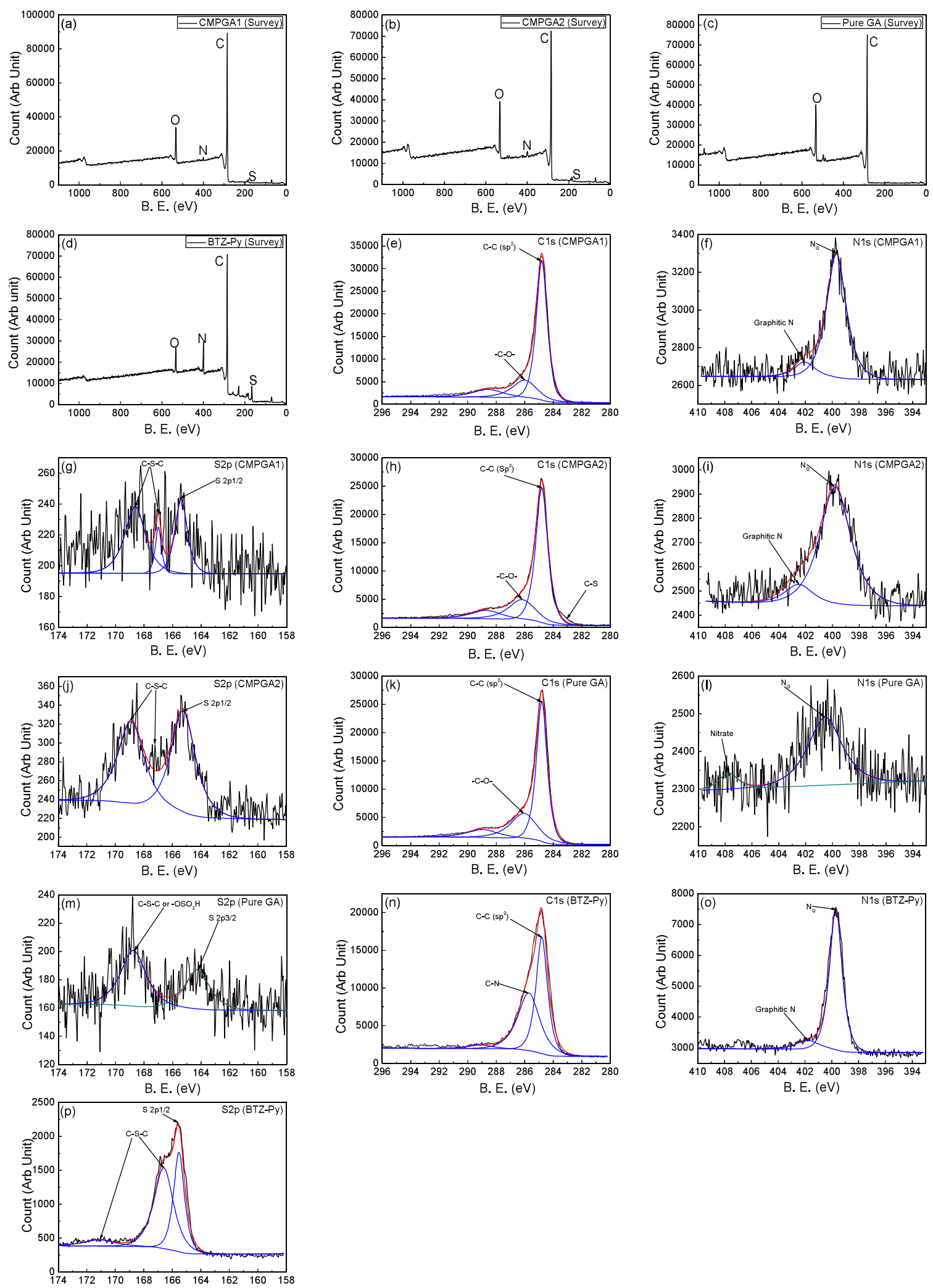

Fig 6 B. E. $(\mathrm{eV})$ 

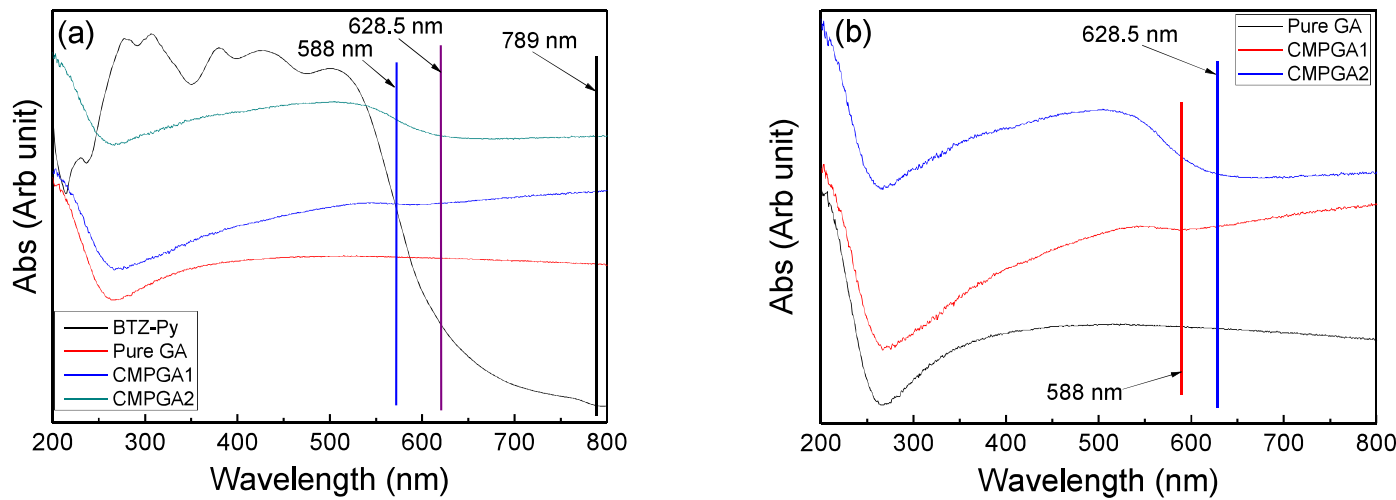

Fig 7 

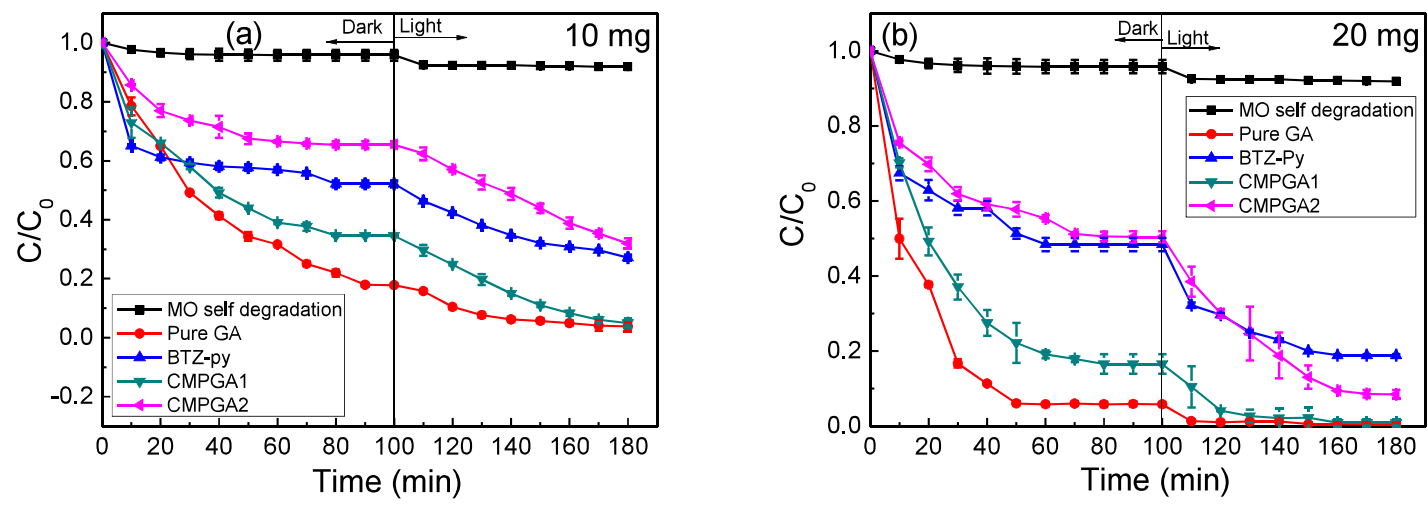

Fig 8 

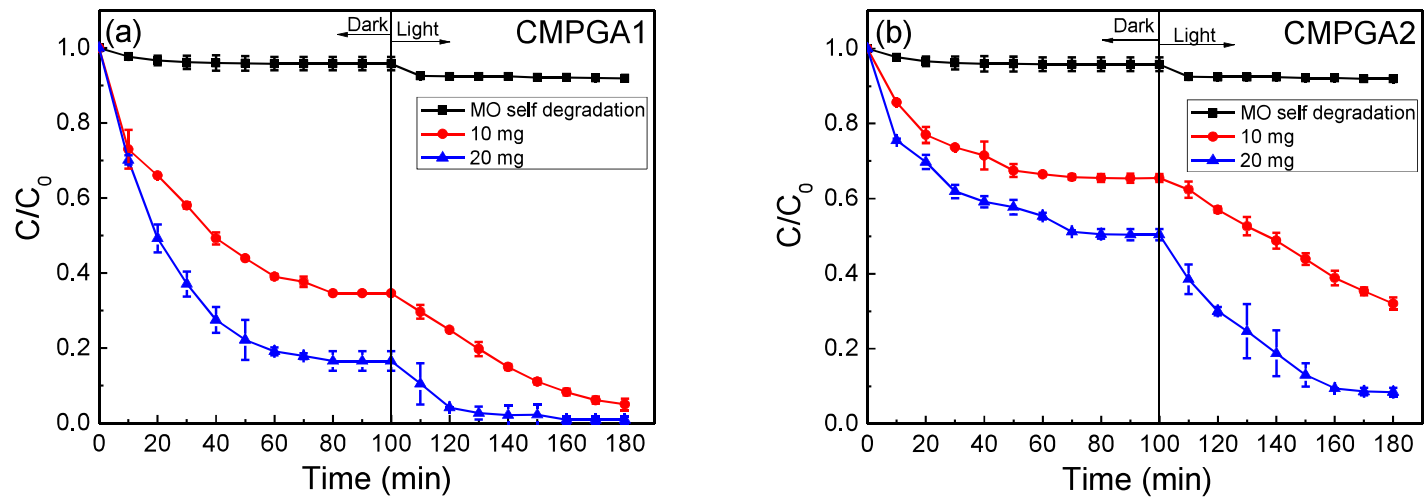

Fig 9 


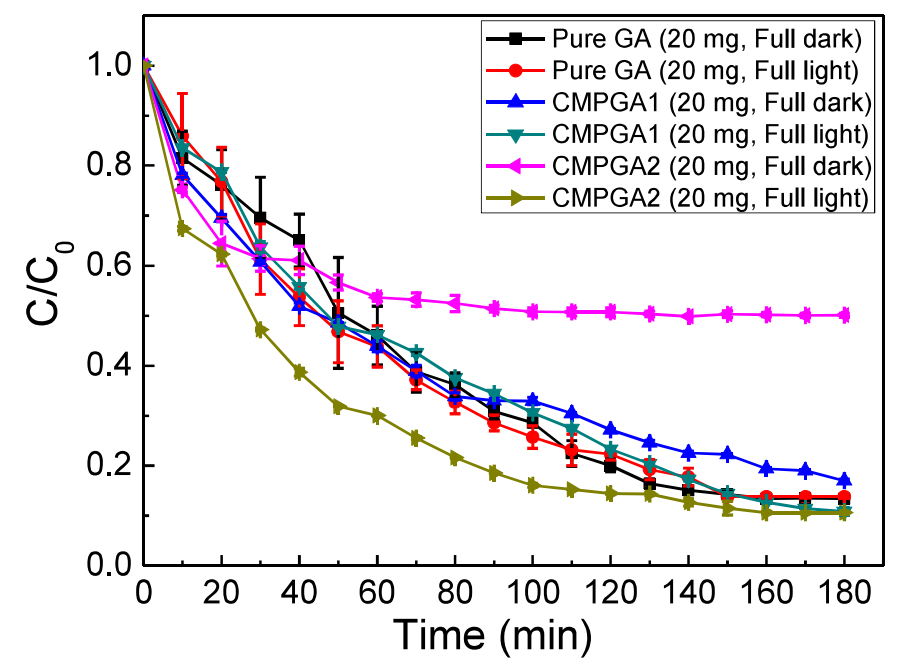

Fig 10 

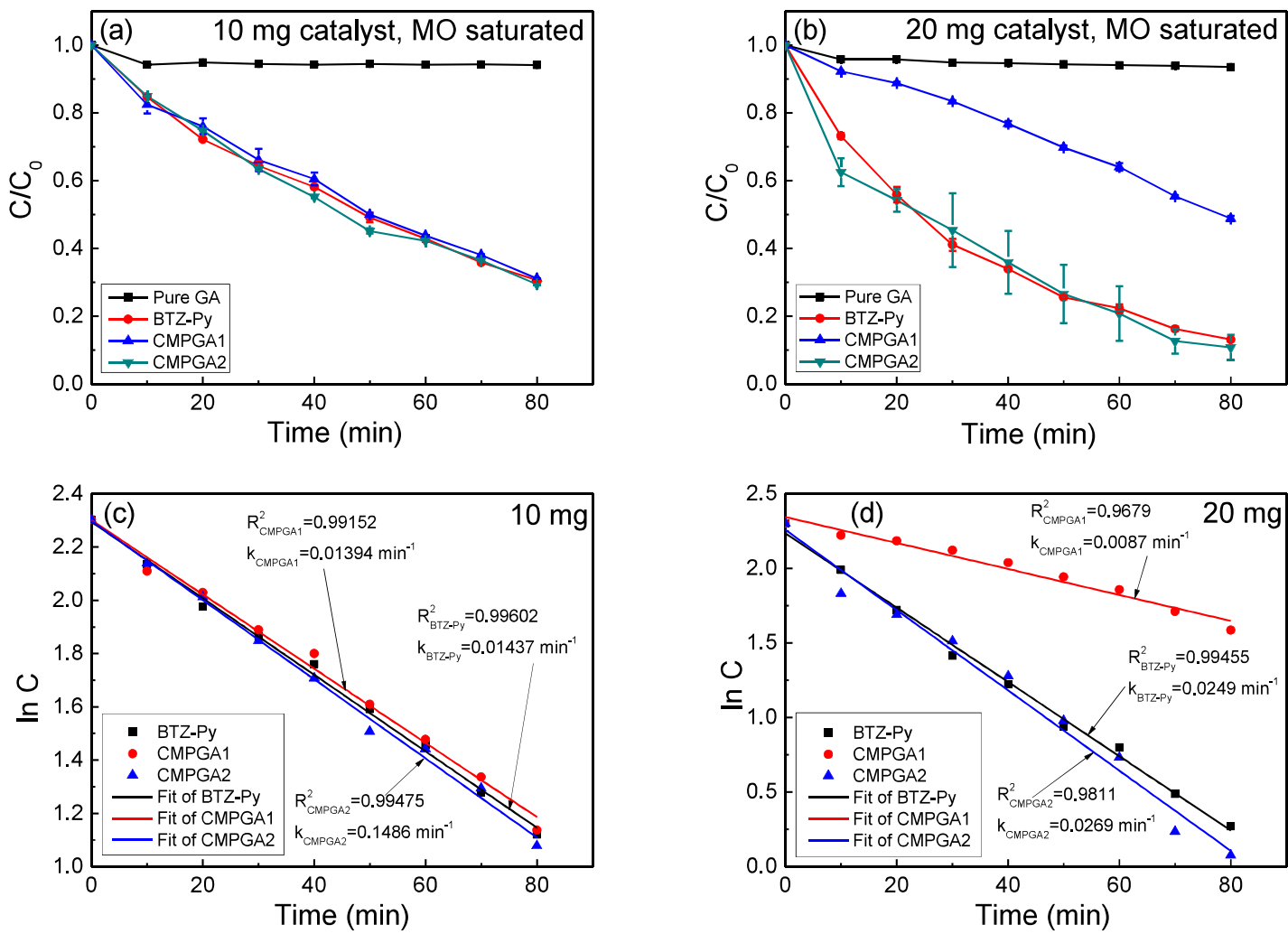

Fig 11 


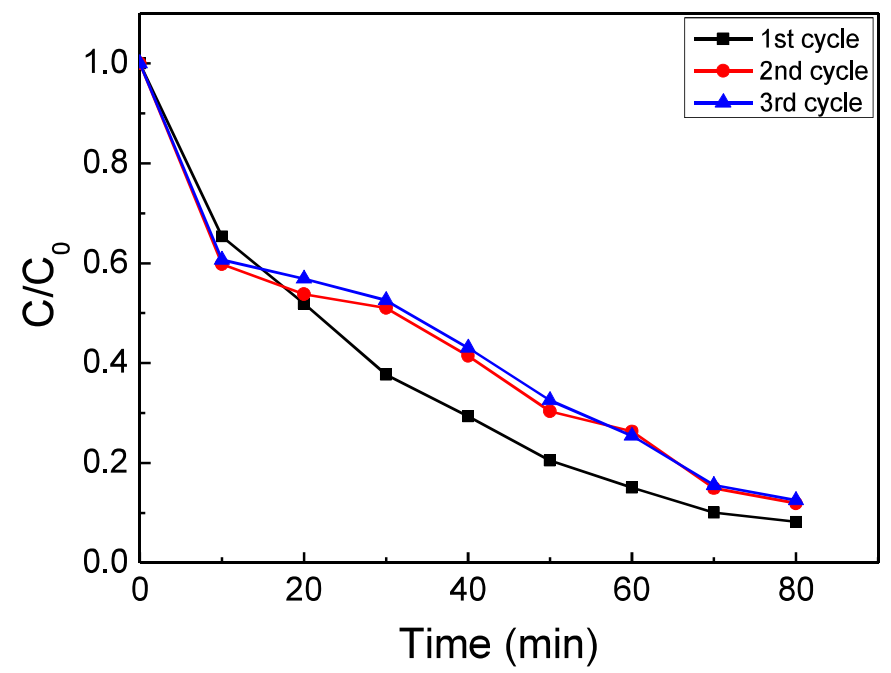

Fig 12. 
Table 1. Elemental information of the $\mathrm{C}$, $\mathrm{O}$, and $\mathrm{S}$ composition in CMPGA samples vs pure GA based on EDX analysis (at $\%$ and $w t \%$ based).

\begin{tabular}{llll}
\hline Sample & $\mathrm{C}(\mathrm{at} \%, \mathrm{wt} \%)$ & $\mathrm{O}(\mathrm{at} \%, \mathrm{wt} \%)$ & $\mathrm{S}(\mathrm{at} \%, \mathrm{wt} \%)$ \\
\hline 0:1 (BTZ-Py:GO $\left.\left(3 \mathrm{mg} \mathrm{ml}^{-1}\right)\right)($ Pure GA) & $69.79,58.41$ & $22.81,25.43$ & 0,0 \\
2:5 (BTZ-Py:GO $\left.\left(3 \mathrm{mg} \mathrm{ml}^{-1}\right)\right)($ CMPGA1) & $83.41,78.25$ & $15.79,19.73$ & $0.81,2.02$ \\
1:1 (BTZ-Py:GO $\left(3 \mathrm{mg} \mathrm{ml}^{-1}\right)$ ) (CMPGA2) & $84.09,77.93$ & $13.94,17.21$ & $1.97,4.86$ \\
\hline
\end{tabular}

Table 2. Activity of the CMPGAs vs pure GA $(20 \mathrm{mg})$ in the fully dark and full light on MO photodecomposition (3h).

\begin{tabular}{lllll}
\hline Sample & Environment & $\mathrm{MO}(\mathrm{ppm})$ & $\mathrm{V}_{\mathrm{MO}}(\mathrm{ml})$ & Efficiency \% \\
\hline CMPGA2 (Polymer=1 mg) & Dark & 10 & 40 & 50.0 \\
CMPGA2 (Polymer=1 mg) & Visible-light & 10 & 40 & 90.4 \\
CMPGA1 (Polymer=0.4 mg) & Dark & 10 & 40 & 83.0 \\
CMPGA1 (Polymer=0.4 mg) & Visible-light & 10 & 40 & 89.1 \\
Pure GA (Polymer=0 mg) & Dark & 10 & 40 & 86.6 \\
Pure GA (Polymer=0 mg) & Visible-light & 10 & 40 & 86.2 \\
\hline
\end{tabular}

Table 3. Activity of the representative GA based catalyst in the vis-light driven MO photodecomposition.

\begin{tabular}{|c|c|c|c|c|c|c|c|}
\hline \multicolumn{2}{|l|}{ Sample } & $\begin{array}{l}\text { Amount of catalyst } \\
(\mathrm{mg})\end{array}$ & $\begin{array}{l}\mathrm{MO} \\
(\mathrm{ppm})\end{array}$ & $\begin{array}{l}\mathrm{V}_{\mathrm{MO}} \\
(\mathrm{ml})\end{array}$ & $\begin{array}{l}\text { Time } \\
(\mathrm{min})\end{array}$ & $\begin{array}{l}\text { Efficiency } \\
\%\end{array}$ & Ref \\
\hline \multicolumn{2}{|l|}{ CNGA-2 } & $30\left(\mathrm{gC}_{3} \mathrm{~N}_{4}=20 \mathrm{mg}\right)$ & 20 & 30 & 240 & 94 & {$[1]$} \\
\hline \multicolumn{2}{|l|}{ BiOBr-G10 } & $100(\mathrm{BiOBr}=10 \mathrm{mg})$ & 10 & 100 & 120 & 94.7 & [3] \\
\hline \multicolumn{2}{|l|}{ 9-CN/GOA } & $\mathrm{N} / \mathrm{A}$ & 20 & 50 & 40 & 91.1 & [14] \\
\hline \multicolumn{2}{|l|}{ 40-NOG } & $50\left(\mathrm{Cu}_{2} \mathrm{O}=20 \mathrm{mg}\right)$ & 30 & 100 & 120 & 96 & {$[15]$} \\
\hline \multicolumn{2}{|c|}{$\mathrm{Cu}_{2} \mathrm{O} / \mathrm{RGO}$ aerogel } & $60\left(\mathrm{Cu}_{2} \mathrm{O}=22.2 \mathrm{mg}\right)$ & 5 & 60 & 600 & 70 & [8] \\
\hline \multicolumn{2}{|l|}{ CMPGA1 } & $10($ Polymer $=0.2 \mathrm{mg})$ & 10 & 40 & 180 & 95 & This work \\
\hline \multicolumn{2}{|l|}{ CMPGA1 } & 20 (Polymer $=0.4 \mathrm{mg})$ & 10 & 40 & 180 & 68 & This work \\
\hline & $10($ Polymer $=0.5 \mathrm{mg})$ & 10 & 40 & 180 & 99.1 & This work \\
\hline \multicolumn{2}{|l|}{ CMPGA2 } & $20($ Polymer $=1 \mathrm{mg})$ & 10 & 40 & 180 & 91.6 & This work \\
\hline \multicolumn{2}{|l|}{$\begin{array}{l}\text { CMPGA1 } \\
\text { saturated) }\end{array}$} & $10($ Polymer $=0.2 \mathrm{mg})$ & 10 & 40 & 80 & 68.9 & This work \\
\hline \multicolumn{2}{|l|}{$\begin{array}{l}\text { CMPGA1 } \\
\text { saturated) }\end{array}$} & $20($ Polymer $=0.4 \mathrm{mg})$ & 10 & 40 & 80 & 51.2 & This work \\
\hline \multicolumn{2}{|l|}{$\begin{array}{l}\text { CMPGA2 } \\
\text { saturated) }\end{array}$} & $10($ Polymer $=0.5 \mathrm{mg})$ & 10 & 40 & 80 & 70.7 & This work \\
\hline $\begin{array}{l}\text { CMPGA2 } \\
\text { saturated) }\end{array}$ & (MO- & 20 (Polymer=1 mg) & 10 & 40 & 80 & 89.2 & This work \\
\hline
\end{tabular}


Table 4. Activity of the representative GA based catalyst in the vis-light driven MO photodecomposition at 60th min after the light on reaction started.

\begin{tabular}{|c|c|c|c|c|c|c|}
\hline \multicolumn{2}{|l|}{ Sample } & Amount (mg) & MO (ppm) & $\mathrm{V}_{\mathrm{MO}}(\mathrm{ml})$ & Efficiency \% & Ref \\
\hline \multicolumn{2}{|l|}{ CNGA-2 } & $30\left(\mathrm{gC}_{3} \mathrm{~N}_{4}=20 \mathrm{mg}\right)$ & 20 & 30 & 65 & [1] \\
\hline \multicolumn{2}{|c|}{ BiOBBr-G10 } & $100(\mathrm{BiOBr}=10 \mathrm{mg})$ & 10 & 100 & 84 & [3] \\
\hline \multicolumn{2}{|c|}{$40-\mathrm{NOG}$} & $50\left(\mathrm{Cu}_{2} \mathrm{O}=20 \mathrm{mg}\right)$ & 30 & 100 & 70 & [15] \\
\hline \multicolumn{2}{|c|}{$\mathrm{Cu}_{2} \mathrm{O} / \mathrm{RGO}$ aerogel } & $60\left(\mathrm{Cu}_{2} \mathrm{O}=22.2 \mathrm{mg}\right)$ & 5 & 60 & 20 & [8] \\
\hline \multicolumn{2}{|l|}{ CMPGA1 } & $10($ Polymer $=0.2 \mathrm{mg})$ & 10 & 40 & 91.8 & This work \\
\hline \multicolumn{2}{|l|}{ CMPGA2 } & $10($ Polymer $=0.5 \mathrm{mg})$ & 10 & 40 & 61.2 & This work \\
\hline \multicolumn{2}{|l|}{ CMPGA1 } & 20 (Polymer=0.4 mg) & 10 & 40 & 99.1 & This work \\
\hline \multicolumn{2}{|l|}{ CMPGA2 } & $20($ Polymer $=1 \mathrm{mg})$ & 10 & 40 & 90.5 & This work \\
\hline \multicolumn{7}{|c|}{ (IVU- 10 (Polymer $=0.2 \mathrm{mg})$} \\
\hline $\begin{array}{l}\text { CMPGA2 } \\
\text { saturated) }\end{array}$ & (MO- & $10($ Polymer $=0.5 \mathrm{mg})$ & 10 & 40 & 57.7 & This work \\
\hline $\begin{array}{l}\text { CMPGA1 } \\
\text { saturated) }\end{array}$ & $(\mathrm{MO}-$ & $20($ Polymer $=0.4 \mathrm{mg})$ & 10 & 40 & 35.9 & This work \\
\hline $\begin{array}{l}\text { CMPGA2 } \\
\text { saturated) }\end{array}$ & (MO- & 20 (Polymer $=1 \mathrm{mg}$ ) & 10 & 40 & 79.2 & This work \\
\hline
\end{tabular}

